\title{
A Topos Foundation for Theories of Physics: II. Daseinisation and the Liberation of Quantum Theory
}

\author{
A. Döring 1 \\ and \\ C.J. Isham ${ }^{2}$ \\ The Blackett Laboratory \\ Imperial College of Science, Technology \& Medicine \\ South Kensington \\ London SW7 2BZ
}

6 March, 2007

\begin{abstract}
This paper is the second in a series whose goal is to develop a fundamentally new way of constructing theories of physics. The motivation comes from a desire to address certain deep issues that arise when contemplating quantum theories of space and time.

Our basic contention is that constructing a theory of physics is equivalent to finding a representation in a topos of a certain formal language that is attached to the system. Classical physics arises when the topos is the category of sets. Other types of theory employ a different topos.

In this paper, we study in depth the topos representation of the propositional language, $\mathcal{P} \mathcal{L}(S)$, for the case of quantum theory. In doing so, we make a direct link with, and clarify, the earlier work on applying topos theory to quantum physics. The key step is a process we term 'daseinisation' by which a projection operator is mapped to a sub-object of the spectral presheaf - the topos quantum analogue of a classical state space.

In the second part of the paper we change gear with the introduction of the more sophisticated local language $\mathcal{L}(S)$. From this point forward, throughout the rest of the series of papers, our attention will be devoted almost entirely to this language. In the present paper, we use $\mathcal{L}(S)$ to study 'truth objects' in the topos. These are objects in the topos that play the role of states: a necessary development as the spectral presheaf has no global elements, and hence there are no microstates in the sense of classical physics. Truth objects therefore play a crucial role in our formalism.
\end{abstract}

\footnotetext{
${ }^{1}$ email: a.doering@imperial.ac.uk

${ }^{2}$ email: c.isham@imperial.ac.uk
} 


\section{Introduction}

This is the second in a series of papers whose aim is to formulate a general framework for expressing theories of physics in a topos other than that of sets. In paper I we introduced the idea that a formal language can be attached to each system, $S$, and that, in the broadest sense, constructing a physical theory of $S$ is equivalent to finding a representation, $\phi$, of this language in a topos $\tau_{\phi}[1]$.

It is expected that, for a given system, different theory-types (such as classical physics, quantum physics, and others yet to be discovered) will be represented in different topoi. Typically, more than one system will share the same language, and, for a given theory-type, these systems will generally be represented in the same topos. However, the details of the representation will be system dependent.

For example, let the system, $S$, be a non-relativistic point particle moving in three dimensions with the Hamiltonian $H=\underline{p} \cdot \underline{p} / 2 m^{2}+V(\underline{x})$. The application of the theory-type "classical physics" involves a representation in the topos, Sets, of sets, and different representations of the language of $S$ correspond to different choices of the potential $V(\underline{x})$. On the other hand, a representation of the same system, but for the theory-type "quantum physics", employs a topos of presheaves, Sets ${ }^{C^{\circ p}}$, over a certain category $\mathcal{C}$ (see below) that depends on the system. Once again, different Hamiltonians correspond to different representations in this topos.

In the first paper, I, we discussed two different types of language that can be attached to a system $S$. The first is a simple propositional language, $\mathcal{P} \mathcal{L}(S)$, that is generated by primitive propositions of the form " $A \varepsilon \Delta$ ". Such a proposition is to be understood in a realist fashion as asserting "The physical quantity $A$ has a value that lies in the (Borel) subset $\Delta$ of $\mathbb{R}$ ". The language $\mathcal{P} \mathcal{L}(S)$ has the logical connectives $\wedge, \vee, \neg, \Rightarrow$; but nothing else. In particular, it does not include the quantifiers $\forall$ and $\exists$. Nevertheless, it does enable compound propositions about the world to be asserted. It also has a deductive structure that follows from a set of axioms that ensure the validity of intuitionistic logic. The choice of intuitionistic logic over against Boolean logic is made with the hindsight of knowing that each topos has an internal logic of this type.

This propositional language is used in the first half of the present paper where we explore in detail the representation of propositions by (clopen) sub-objects of the state object (the significance of the word 'clopen' is explained below). However, for the sake of overall clarity it should be emphasised that the work involving $\mathcal{P} \mathcal{L}(S)$ is a something of a side-line to our main programme, which is concerned with the local language $\mathcal{L}(S)$. In fact, logically speaking, the reader could jump straight to Section 4, which deals with the idea of a 'truth object' in the context of the language $\mathcal{L}(S)$. However, we decided to include the $\mathcal{P} \mathcal{L}(S)$-material because it is what links most closely to the original work on using topos ideas in quantum theory. Indeed, as we shall see, the new material on the representation of $\mathcal{P} \mathcal{L}(S)$ includes a vital concept that was not understood before, and which places the earlier work in a much clearer light.

The second language introduced in paper I is far more powerful. Firstly, it is higher 
order, so that the existential connectives $\forall, \exists$ are included. Secondly, this, so-called, 'local' language, $\mathcal{L}(S)$, is typed: a feature that allows the most important ingredients of any theory of physics to be included in a very specific way.

Specifically, $\mathcal{L}(S)$ contains the 'ground-type symbols', $\Sigma$ and $\mathcal{R}$, which are construed as the 'linguistic precursors' of the state object, and quantity-value object, respectively. Thus, in any representation $3, \phi$, of $\mathcal{L}(S)$ in a topos $\tau_{\phi}$, the symbols $\Sigma$ and $\mathcal{R}$ are mapped to objects $\Sigma_{\phi}$ and $\mathcal{R}_{\phi}$ in $\tau_{\phi}$ which are the state object and quantity-value object, respectively, for this particular theory.

The application of this structure to classical physics was discussed in I, and in the present paper, we want to turn to the, more challenging, case of quantum theory. We know from the earlier work on applying topos ideas to quantum theory, that the topos of the quantum representation, $\phi$, of $\mathcal{L}(S)$ is the category of presheaves, $\operatorname{Sets}^{\mathcal{V}(\mathcal{H})^{\mathrm{op}}}$, over the category $\mathcal{V}(\mathcal{H})$ of unital, abelian von Neumann subalgebras of $\mathcal{B}(\mathcal{H})$. Here, $\mathcal{B}(\mathcal{H})$ is the non-commutative algebra of all bounded operators on the Hilbert space, $\mathcal{H}$, of the quantum system. In this representation, the state object, $\Sigma_{\phi}$, is the spectral presheat $\underline{\Sigma}$ that was discussed at length in the earlier work [13, 14, 15, 16]; see Definition 2.3 in this paper. This is the quantum analogue of the classical state space.

Still, it remains true that only a limited range of questions can be addressed using the propositional language, $\mathcal{P} \mathcal{L}(S)$, and to appreciate properly the full scope of the 'toposification' ideas it is essential to employ the more sophisticated local language $\mathcal{L}(S)$. For this reason, in the second part of the paper we switch to using $\mathcal{L}(S)$, and this will be the focus of our attention for the remaining papers in this series.

Amongst other things, using $\mathcal{L}(S)$ involves identifying the quantity-value presheaf: something that is not part of the simpler language $\mathcal{P} \mathcal{L}(S)$. The quantity-value presheaf, $\mathcal{R}_{\phi}$, and related topics are discussed in papers III and IV [2, 3]. In particular, in paper III we show how a function symbol $A: \Sigma \rightarrow \mathcal{R}$ (i.e., a physical quantity) can be represented by an arrow $A_{\phi}: \Sigma_{\phi} \rightarrow \mathcal{R}_{\phi}$, where $\Sigma_{\phi}$ is the spectral presheaf $\underline{\Sigma}$.

Returning to $\mathcal{P} \mathcal{L}(S)$, the plan of the present paper is as follows. A key step in constructing a topos representation is an operation that we call 'daseinisation', and this is discussed in detail in Section 2. This involves constructing a map from the lattice of projectors $\mathcal{P}(\mathcal{H})$ to the Heyting algebra $\operatorname{Sub}_{\mathrm{cl}}(\underline{\Sigma})$ of clopen sub-objects of the spectral presheaf $\underline{\Sigma}$. The motivation for this construction lies in the earlier work on topos theory and quantum mechanics [13, 14, 15, 16] but the key technical ingredient is due to de Groote [10].

One feature of this construction is that there are more clopen sub-objects of $\underline{\Sigma}$ than those given by daseinisation of projection operators. This raises the question of

\footnotetext{
${ }^{3}$ A more comprehensive notation is $\tau_{\phi}(S)$, which draws attention to the system $S$ under discussion; similarly, the state object could be written as $\Sigma_{\phi, S}$, and so on. This extended notation is used in paper IV where we are concerned with the relations between different systems, and then it is essential to indicate which system is meant. However, in the present paper, only one system at a time is being considered, and so the truncated notation is adequate.

${ }^{4}$ As a pedagogical aid, all presheaves will be denoted with an underlined symbol, like $\underline{\Sigma}, \underline{\mathcal{R}}, \underline{\Omega}$ etc.
} 
whether there are special, definitive features of the sub-objects that are of the form $\delta(\hat{P}), \hat{P} \in \mathcal{P}(\mathcal{H})$, and this is discussed in detail in Section 3 where we show that the daseinised propositions are 'optimal' in the lattice of all sub-objects of $\underline{\Sigma}$.

The state object, $\underline{\Sigma}$, in the quantum topos has no global elements (this statement is equivalent to the Kochen-Specker theorem) and, therefore, the assignation of 'truth values' to propositions needs an approach that is structurally different from the one used in classical physics. This issue is discussed in detail in Section 4, using a method that rests heavily on the local language $\mathcal{L}(S)$. In particular, we introduce into $\mathcal{L}(S)$ a variable that is the linguistic precursor of the truth object needed in the topos representations. We then use this to motivate an analogous construction in quantum theory for the propositional language $\mathcal{P} \mathcal{L}(S)$. The same kind of truth object is used in the context of a representation of the local language $\mathcal{L}(S)$ in quantum theory, see paper III.

A cautionary caveat. At this point, before embarking on the main text, we want to remark on the scope of the work reported in this paper, and in paper III: i.e., the application of topos ideas to standard quantum theory via the representation of a system language.

For the language $\mathcal{P} \mathcal{L}(S)$, a key result from the topos constructions is that, given any quantum state $|\psi\rangle$, there are generalised truth values, $\nu(A \varepsilon \Delta ;|\psi\rangle)$, for propositions " $A \varepsilon \Delta$ ". These truth values belong to the Heyting algebra, $\Gamma \Omega_{\phi}$, of global elements of the sub-object classifier, $\Omega_{\phi}$, of the topos concerned. In making these assignments, nothing is said about 'measurements', or 'observers', or even 'probability': there is just the truth value $\nu(A \varepsilon \Delta ;|\psi\rangle) \in \Gamma \Omega_{\phi}$.

It is this absence of instrumentalist concepts that motivates/justifies the appellation 'neo-realist' for this topos approach to quantum theory. However, we want to insert a cautionary remark. Our specific topos constructions for quantum theory are based on the usual mathematical formalism of self-adjoint operators on a Hilbert space; and some physicists, such as Bohr, have asserted that this formalism is fundamentally associated with an instrumentalist interpretation of the theory, whose predictions are probabilities of the results of making measurements. If this is true, our neo-realism would sit uncomfortably on the formalism.

On the other hand, maybe our quantum results are meaningful as they standparticularly the ones obtained using the local language $\mathcal{L}(S)$ (in paper III). If so, an intriguing challenge would be to use these methods to construct a neo-realist perspective on a quantum-cosmological model. A particularly appropriate example is the recent construction by Kessari of a ( $\mathrm{HPO}^{5}$ ) consistent-histories version of the RobertsonWalker universe [4].

Evidently, the operation we have called 'daseinisation' is a bridge between the

\footnotetext{
${ }^{5}$ There are various mathematical approaches to formulating consistent-histories quantum theory, but the one to which our topos work could most easily be adapted is the 'History Projection Operator' (HPO) scheme in which history propositions are represented by projection operators.
} 
intrinsic instrumentalism of the standard quantum formalism, and a full neo-realism associated with the language $\mathcal{L}(S)$ which makes no reference to entities outside that language. In this sense, standard quantum theory is a 'hybrid' object that interpolates between the instrumentalist world, with its various background structures, and the neo-realist world, in which almost everything has a linguistic precursor.

However, as we keep emphasising, our main goal is to construct tools for developing new types of theory, not just to look at standard quantum theory from a novel angle. For us, the most important thing about the topos approach to standard quantum theory is that (we hope) it serves as a paradigmatic example of the types of mathematical structure that might be needed to develop theories that go 'beyond' standard quantum theory, and that are not slaves to the use of continuum entities. We will have more to say about this central theme at various points in this series of papers.

\section{Quantum Propositions as Sub-Objects of the Spec- tral Presheaf}

\subsection{From Projections to Global Sections of the Outer Presheaf}

\subsubsection{The Definition of $\delta(\hat{P})_{V}$}

The fundamental thesis of our work is that, in constructing theories of physics, one should seek representations of a formal language in a topos that may be other than Sets. We want now to study this idea closely in the context of the toposification of standard quantum theory, with particular emphasis on a topos representation of propositions. Most 'standard' quantum systems (for example, one-dimensional motion with a Hamiltonian $\left.H=\frac{p^{2}}{2 m}+V(x)\right)$ are obtained by 'quantising' a classical system, and consequently the language is the same as it is for the classical system.

As explained in the Introduction, in the first half of this paper we concentrate on the propositional language $\mathcal{P} \mathcal{L}(S)$, so that the critical task is to find the map $\pi_{\mathrm{qt}}: \mathcal{P} \mathcal{L}(S)_{0} \rightarrow \operatorname{Sub}_{\mathrm{cl}}(\underline{\Sigma})$, where the primitive propositions in $\mathcal{P} \mathcal{L}(S)_{0}$ are of the form " $A \varepsilon \Delta "$ " (the notation is explained in paper I [1]). As we shall see, this is where the critical concept of daseinisation arises: the procedure whereby a projector $\hat{P}$ is transformed to a (clopen) sub-object, $\delta(\hat{P})$, of the spectral presheaf in the topos $\operatorname{Sets}{ }^{\mathcal{V}(\mathcal{H})^{\mathrm{op}}}$.

In standard quantum theory, a physical quantity is represented by a self-adjoint operator $\hat{A}$ in the algebra, $\mathcal{B}(\mathcal{H})$, of all bounded operators on $\mathcal{H}$. If $\Delta \subseteq \mathbb{R}$ is a Borel subset, we know from the spectral theorem that the proposition " $A \varepsilon \Delta$ " is represented by ${ }^{6}$ a projection operator $\hat{E}[A \in \Delta]$ in $\mathcal{B}(\mathcal{H})$. For typographical simplicity, for the rest of this Section, $\hat{E}[A \in \Delta]$ will be denoted by $\hat{P}$.

\footnotetext{
${ }^{6}$ Note, however, that the map from propositions to projections is not injective: two propositions " $A \varepsilon \Delta_{1}$ " and " $B \varepsilon \Delta_{2}$ " about two distinct physical quantities, $A$ and $B$, can be represented by the same projector: i.e., $\hat{E}\left[A \in \Delta_{1}\right]=\hat{E}\left[B \in \Delta_{2}\right]$.
} 
We are going to consider the projection operator $\hat{P}$ from the perspective of the 'category of contexts' that is the basis of the topos approach to quantum theory. There are several possible choices for this category, and these are considered in detail in the original papers [13, 14, 15, 16]. Mathematically, they are ultimately all equivalent, but here we have chosen to use the category $\mathcal{V}(\mathcal{H})$ of unital, abelian subalgebras of $\mathcal{B}(\mathcal{H})$. The category structure is that of a partially-ordered set whose objects are the abelian subalgebras, and in which there is an arrow $i_{V^{\prime} V}: V^{\prime} \rightarrow V, V^{\prime}, V \in \mathrm{Ob}(\mathcal{V}(\mathcal{H})) .7$ if and only if $V^{\prime} \subseteq V$. By definition, the trivial subalgebra $V_{0}=\mathbb{C} \hat{1}$ is not included in the objects of $\mathcal{V}(\mathcal{H})$. A context could also be called a 'world-view', or a 'window on the world', or even a Weltanschauung8; mathematicians call it a 'stage of truth'.

The critical question is what can be said about the projector $\hat{P}$ 'from the viewpoint' of a particular context $V \in \mathrm{Ob}(\mathcal{V}(\mathcal{H}))$ ? If $\hat{P}$ belongs to $V$ then a 'full' image of $\hat{P}$ is obtained from this view-point, and there is nothing more to say. However, suppose the abelian subalgebra $V$ does not contain $\hat{P}$ : what then?

We need to 'approximate' $\hat{P}$ from the perspective of $V$, and an important ingredient in our work is to define this as meaning the 'smallest' projection operator, $\delta(\hat{P})_{V}$, in $V$ that is greater than, or equal to, $\hat{P}$ :

$$
\delta(\hat{P})_{V}:=\bigwedge\{\hat{Q} \in \mathcal{P}(V) \mid \hat{Q} \succeq \hat{P}\} .
$$

where ' $\succeq$ ' is the usual 'quantum-logic' ordering of projection operators, and where $\mathcal{P}(V)$ denotes the set of all projection operators in $V$.

To see what this means, let $\hat{P}$ and $\hat{Q}$ represent the propositions " $A \varepsilon \Delta$ " and " $A \varepsilon \Delta$ " respectively with $\Delta \subseteq \Delta^{\prime}$, so that $\hat{P} \preceq \hat{Q}$. Since we learn less about the value of $A$ from the proposition " $A \varepsilon \Delta^{\prime \prime}$ " than from " $A \varepsilon \Delta^{\prime}$ ", the former proposition is said to be weaker. Clearly, the weaker proposition " $A \varepsilon \Delta^{\prime \prime}$ " is implied by the stronger proposition " $A \varepsilon \Delta$ ". The construction of $\delta(\hat{P})_{V}$ as the smallest projection in $V$ greater than or equal to $\hat{P}$ thus gives the strongest proposition expressible in $V$ that is implied by $\hat{P}$ (although, if $\hat{A} \notin V$, the projection $\delta(\hat{P})_{V}$ cannot be interpreted as a proposition about $A$ in general) 9 Note that if $\hat{P}$ belongs to $V$, then $\delta(\hat{P})_{V}=\hat{P}$. The mapping $\hat{P} \mapsto \delta(\hat{P})_{V}$ was originally introduced by de Groote in [10], who called it the ' $V$-support' of $\hat{P}$.

The key idea in this part of our scheme is that rather than thinking of a quantum proposition, " $A \varepsilon \Delta$ ", as being represented by a single projection operator $\hat{E}[A \in \Delta]$, we instead consider the collection $\left\{\delta(\hat{E}[A \in \Delta])_{V} \mid V \in \mathrm{Ob}(\mathcal{V}(\mathcal{H}))\right\}$ of one projection operator for each context $V$. As we will see, the link with topos theory is that this collection of projectors is a global element of a certain presheaf.

This 'certain' presheaf is in fact the 'outer' presheaf, which is defined as follows:

\footnotetext{
${ }^{7}$ We denote by $\operatorname{Ob}(\mathcal{C})$ the collection of all objects in the category $\mathcal{C}$.

${ }^{8}$ 'Weltanschauung' is a splendid German word. 'Welt' means world; 'schauen' is a verb and means to look, to view; 'anschauen' is to look at; and '-ung' at the end of a word can make a noun from a verb. So it's Welt-an-schau-ung.

${ }^{9}$ Note that we use the fact that the lattice $\mathcal{P}(V)$ of projections in $V$ is complete. This is the main reason why we chose von Neumann subalgebras rather than $C^{*}$-algebras: the former contain enough projections, and their projection lattices are complete.
} 
Definition 2.1 The outer 10 presheaf $\underline{O}$ is defined over the category $\mathcal{V}(\mathcal{H})$ as follows [13, 15]:

(i) On objects $V \in \mathrm{Ob}(\mathcal{V}(\mathcal{H})): \underline{O}_{V}:=\mathcal{P}(V)$

(ii) On morphisms $i_{V^{\prime} V}: V^{\prime} \subseteq V$ : The mapping $\underline{O}\left(i_{V^{\prime} V}\right): \underline{O}_{V} \rightarrow \underline{O}_{V^{\prime}}$ is given by $\underline{O}\left(i_{V^{\prime} V}\right)(\hat{\alpha}):=\delta(\hat{\alpha})_{V^{\prime}}$ for all $\hat{\alpha} \in \mathcal{P}(V)$.

With this definition, it is clear that the assignment $V \mapsto \delta(\hat{P})_{V}$ defines a global element of the presheaf $\underline{O}$. Indeed, for each context $V$, we have the projector $\delta(\hat{P})_{V} \in$ $\mathcal{P}(V)=\underline{O}_{V}$, and if $i_{V^{\prime} V}: V^{\prime} \subseteq V$, then

$$
\delta\left(\delta(\hat{P})_{V}\right)_{V^{\prime}}=\bigwedge\left\{\hat{Q} \in \mathcal{P}\left(V^{\prime}\right) \mid \hat{Q} \succeq \delta(\hat{P})_{V}\right\}=\delta(\hat{P})_{V^{\prime}}
$$

and so the elements $\delta(\hat{P})_{V}, V \in \mathrm{Ob}(\mathcal{V}(\mathcal{H}))$, are compatible with the structure of the outer presheaf. Thus we have a mapping

$$
\begin{aligned}
\delta: \mathcal{P}(\mathcal{H}) & \rightarrow \Gamma \underline{O} \\
\hat{P} & \mapsto\left\{\delta(\hat{P})_{V} \mid V \in \mathrm{Ob}(\mathcal{V}(\mathcal{H}))\right\}
\end{aligned}
$$

from the projectors in $\mathcal{P}(\mathcal{H})$ to the global elements, $\Gamma \underline{O}$, of the outer presheaf 11

\subsubsection{Properties of the Mapping $\delta: \mathcal{P}(\mathcal{H}) \rightarrow \Gamma \underline{O}$.}

Let us now note some properties of the map $\delta: \mathcal{P}(\mathcal{H}) \rightarrow \Gamma \underline{O}$ that are relevant to our overall scheme.

1. For all contexts $V$, we have $\delta(\hat{0})_{V}=\hat{0}$, i.e., the null projector $\hat{0}$ is mapped to the null element of $\underline{O}$.

The null projector represents all propositions of the form " $A \varepsilon \Delta$ " with the property that $\operatorname{sp}(\hat{A}) \cap \Delta=\varnothing$, where $\operatorname{sp}(\hat{A})$ denotes the spectrum of the self-adjoint operator $\hat{A}$. These propositions are trivially false.

2. For all contexts $V$, we have $\delta(\hat{1})_{V}=\hat{1}$, i.e., the unit operator $\hat{1}$ is mapped to the 'unit' element of $\underline{O}$.

The unit operator $\hat{1}$ represents all propositions of the form " $A \varepsilon \Delta$ " with the property that $\operatorname{sp}(\hat{A}) \cap \Delta=\operatorname{sp}(\hat{A})$. These propositions are trivially true.

\footnotetext{
${ }^{10}$ In the original papers by CJI and collaborators, this was called the 'coarse-graining' presheaf, and was denoted $\underline{G}$. The reason for the change of nomenclature will become apparent later.

${ }^{11}$ Vis-a-vis our later work with the language $\mathcal{L}(S)$, we should emphasise that the outer presheaf has no linguistic precursor: in this sense, it has no fundamental status. In fact, we could avoid it altogether and always work directly with the spectral presheaf, $\underline{\Sigma}$ - which, of course, does have a linguistic precursor. However, it is technically useful to introduce the outer presheaf as an intermediate tool.
} 
3. There are global elements of $\underline{O}$ that do not come from a projection operator. This will be discussed later.

However, if $\gamma \in \underline{O}$ is of the form $\delta(\hat{P})$ for some $\hat{P}$, then

$$
\hat{P}=\bigwedge_{V \in \operatorname{Ob}(\mathcal{V}(\mathcal{H}))} \delta(\hat{P})_{V}
$$

because $\delta(\hat{P})_{V} \succeq \hat{P}$ for all $V \in \mathrm{Ob}(\mathcal{V}(\mathcal{H}))$, and $\delta(\hat{P})_{V}=\hat{P}$ for any $V$ that contains $\hat{P}$.

The next result is important as it means that no 'information' is lost in mapping a projection operator $\hat{P}$ to its associated global element, $\delta(\hat{P})$, of the presheaf $\underline{O}$.

Theorem 2.1 The map $\delta: \mathcal{P}(\mathcal{H}) \rightarrow \Gamma \underline{O}$ is injective.

To see this, let $\hat{P}_{1}, \hat{P}_{2}$ be two different projection operators in $\mathcal{P}(\mathcal{H})$. Without loss of generality, let $\hat{P}_{1} \neq \hat{1}$. Let $V_{1}:=\left\{\hat{P}_{1}, \hat{1}\right\}^{\prime \prime}$ denote the abelian von Neumann algebra generated by $\hat{P}_{1}$ and $\hat{1}$. Then $\mathcal{P}\left(V_{1}\right)=\left\{\hat{0}, \hat{P}_{1}, \hat{1}-\hat{P}_{1}, \hat{1}\right\}$. We now run through the possible relations between $\hat{P}_{1}$ and $\hat{P}_{2}$, case by case.

Case (i): $\hat{P}_{1} \prec \hat{P}_{2}$. (Of course, this includes the case $\hat{P}_{2} \prec \hat{P}_{1}$ by simple relabelling.) In this case, $\delta\left(\hat{P}_{1}\right)_{V_{1}}=\bigwedge\left\{\hat{Q} \in \mathcal{P}\left(V_{1}\right) \mid \hat{Q} \succeq \hat{P}_{1}\right\}=\hat{P}_{1}$ and $\delta\left(\hat{P}_{2}\right)_{V_{1}}=\hat{1} \neq \hat{P}_{1}$.

Case (ii): $\hat{1}-\hat{P}_{1} \prec \hat{P}_{2}$. Then $\delta\left(\hat{P}_{1}\right)_{V_{1}}=\hat{P}_{1}$ and

$$
\begin{aligned}
\delta\left(\hat{P}_{2}\right)_{V_{1}} & =\bigwedge\left\{\hat{Q} \in \mathcal{P}\left(V_{1}\right) \mid \hat{Q} \succeq \hat{P}_{2}\right\} \\
& \succeq \bigwedge\left\{\hat{Q} \in \mathcal{P}\left(V_{1}\right) \mid \hat{Q} \succeq \hat{1}-\hat{P}_{1}\right\} \\
& =\hat{1}-\hat{P}_{1}
\end{aligned}
$$

Case (iii): $\hat{P}_{1}=\hat{1}-\hat{P}_{2}$. Then $\delta\left(\hat{P}_{1}\right)_{V_{1}}=\hat{P}_{1}$ and $\delta\left(\hat{P}_{2}\right)_{V_{1}}=\hat{1}-\hat{P}_{1}$.

Case (iv): $\hat{P}_{1} \nprec \hat{P}_{2}$ and $\hat{1}-\hat{P}_{1} \nprec \hat{P}_{2}$. In this case, $\delta\left(\hat{P}_{1}\right)_{V_{1}}=\hat{P}_{1}$ and

$$
\begin{aligned}
\delta\left(\hat{P}_{2}\right)_{V_{1}} & =\bigwedge\left\{\hat{Q} \in \mathcal{P}\left(V_{1}\right) \mid \hat{Q} \succeq \hat{P}_{2}\right\} \\
& =\hat{1}
\end{aligned}
$$

In each case, $\delta\left(\hat{P}_{1}\right)_{V_{1}}=\hat{P}_{1} \neq \delta\left(\hat{P}_{2}\right)_{V_{1}}$, and hence the global elements $\delta\left(\hat{P}_{1}\right)=$ $\left\{\delta\left(\hat{P}_{1}\right)_{V} \mid V \in \mathrm{Ob}(\mathcal{V}(\mathcal{H}))\right\}$ and $\delta\left(\hat{P}_{2}\right)=\left\{\delta\left(\hat{P}_{2}\right)_{V} \mid V \in \mathrm{Ob}(\mathcal{V}(\mathcal{H}))\right\}$ differ.

\subsubsection{A Logical Structure for $\Gamma \underline{O}$ ?}

We have seen that the quantities $\delta(\hat{P}):=\left\{\delta(\hat{P})_{V} \mid V \in \mathrm{Ob}(\mathcal{V}(\mathcal{H}))\right\}, \hat{P} \in \mathcal{P}(\mathcal{H})$, are elements of $\Gamma \underline{O}$, and if they are to represent quantum propositions, one might 
expect/hope that (i) these global elements of $\underline{O}$ form a Heyting algebra; and (ii) this algebra is related in some way to the Heyting algebra of sub-objects of $\underline{\Sigma}$. Let us see how far we can go in this direction.

Our first remark is that any two global elements $\gamma_{1}, \gamma_{2}$ of $\underline{O}$ can be compared at each stage $V$ in the sense of logical implication. More precisely, let $\gamma_{1}(V) \in \mathcal{P}(V)$ denote the $V$ 'th 'component' of $\gamma_{1}$, and ditto for $\gamma_{2}(V)$. Then we have the following result:

Definition 2.2 A partial ordering on $\Gamma \underline{O}$ can be constructed in a 'local' way (i.e., 'local' with respect to the objects in the category $\mathcal{V}(\mathcal{H})$ ) by defining

$$
\gamma_{1} \succeq \gamma_{2} \text { if, and only if, } \forall V \in \mathrm{Ob}(\mathcal{V}(\mathcal{H})), \gamma_{1}(V) \succeq \gamma_{2}(V)
$$

where the ordering on the right hand side of (2.7) is the usual ordering in the lattice of projectors $\mathcal{P}(V)$.

It is trivial to check that (2.7) defines a partial ordering on $\Gamma \underline{\underline{O}}$. Thus $\Gamma \underline{O}$ is a partially ordered set.

Note that if $\hat{P}, \hat{Q}$ are projection operators, then it follows from (2.7) that

$$
\delta(\hat{P}) \succeq \delta(\hat{Q}) \text { if and only if } \hat{P} \succeq \hat{Q}
$$

since $\hat{P} \succeq \hat{Q}$ implies $\delta(\hat{P})_{V} \geq \delta(\hat{Q})_{V}$ for all contexts $V$.12 Thus the mapping $\delta$ : $\mathcal{P}(\mathcal{H}) \rightarrow \Gamma \underline{O}$ preserves the partial order.

The next thing is to see if a logical ' $\vee$ '-operation can be defined on $\Gamma \underline{O}$. Once again, we try a 'local' definition:

Theorem 2.2 A 'V'-structure on $\Gamma \underline{O}$ can be defined locally by

$$
\left(\gamma_{1} \vee \gamma_{2}\right)(V):=\gamma_{1}(V) \vee \gamma_{2}(V)
$$

for all $\gamma_{1}, \gamma_{2} \in \Gamma \underline{O}$, and for all $V \in \mathrm{Ob}(\mathcal{V}(\mathcal{H}))$.

Proof. It is not instantly clear that (2.9) defines a global element of $\underline{O}$. However, a key result in this direction is the following:

Lemma 2.3 For each context $V$, and for all $\hat{\alpha}, \hat{\beta} \in \mathcal{P}(V)$, we have

$$
\underline{O}\left(i_{V^{\prime} V}\right)(\hat{\alpha} \vee \hat{\beta})=\underline{O}\left(i_{V^{\prime} V}\right)(\hat{\alpha}) \vee \underline{O}\left(i_{V^{\prime} V}\right)(\hat{\beta})
$$

for all contexts $V^{\prime}$ such that $V^{\prime} \subseteq V$.

The proof is a straightforward consequence of the definition of the presheaf $\underline{O}$.

\footnotetext{
${ }^{12}$ On the other hand, in general, $\hat{P} \succ \hat{Q}$ does not imply $\delta(\hat{P})_{V} \succ \delta(\hat{Q})_{V}$ but only $\delta(\hat{P})_{V} \succeq \delta(\hat{Q})_{V}$.
} 
One immediate consequence is that (2.9) defines a global element 13 of $\underline{O}$. Hence the theorem is proved.

It is also straightforward to show that, for any pair of projectors $\hat{P}, \hat{Q} \in \mathcal{P}(\mathcal{H})$, we have $\delta(\hat{P} \vee \hat{Q})_{V}=\delta(\hat{P})_{V} \vee \delta(\hat{Q})_{V}$, for all contexts $V \in \mathrm{Ob}(\mathcal{V}(\mathcal{H}))$. This means that, as elements of $\Gamma \underline{O}$,

$$
\delta(\hat{P} \vee \hat{Q})=\delta(\hat{P}) \vee \delta(\hat{Q})
$$

Thus the mapping $\delta: \mathcal{P}(\mathcal{H}) \rightarrow \Gamma \underline{O}$ preserves the logical ' $\vee$ ' operation.

However, there is no analogous equation for the logical ' $\wedge$ '-operation. The obvious local definition would be, for each context $V$,

$$
\left(\gamma_{1} \wedge \gamma_{2}\right)(V):=\gamma_{1}(V) \wedge \gamma_{2}(V)
$$

but this does not define a global element of $\underline{O}$ since, unlike (2.10), for the $\wedge$-operation we have only

$$
\underline{O}\left(i_{V^{\prime} V}\right)(\hat{\alpha} \wedge \hat{\beta}) \preceq \underline{O}\left(i_{V^{\prime} V}\right)(\hat{\alpha}) \wedge \underline{O}\left(i_{V^{\prime} V}\right)(\hat{\beta})
$$

for all $V^{\prime} \subseteq V$. As a consequence, for all $V$, we have only the inequality

$$
\delta(\hat{P} \wedge \hat{Q})_{V} \preceq \delta(\hat{P})_{V} \wedge \delta(\hat{Q})_{V}
$$

and hence

$$
\delta(\hat{P} \wedge \hat{Q}) \preceq \delta(\hat{P}) \wedge \delta(\hat{Q})
$$

It is easy to find examples where the inequality is strict. For example, let $\hat{P} \neq \hat{0}, \hat{1}$ and $\hat{Q}=\hat{1}-\hat{P}$. Then $\hat{P} \wedge \hat{Q}=0$ and hence $\delta_{V}(\hat{P} \wedge \hat{Q})=\hat{0}$, while $\delta(\hat{P})_{V} \wedge \delta(\hat{Q})_{V}$ can be strictly larger than $\hat{0}$, since $\delta(\hat{P})_{V} \succeq \hat{P}$ and $\delta(\hat{Q})_{V} \succeq \hat{Q}$.

\subsubsection{Hyper-Elements of $\Gamma \underline{\underline{O}}$.}

We have seen that the global elements of $\underline{O}$, i.e., the elements of $\Gamma \underline{O}$, can be equipped with a partial-ordering and a ' $\vee$ '-operation, but attempts to define a ' $\wedge$ '-operation in the same way fail because of the inequality in (2.14).

However, the form of (2.13 2.14) suggests the following procedure. Let us define a hyper-element of $\underline{O}$ to be an association, for each stage $V \in \mathrm{Ob}(\mathcal{V}(\mathcal{H}))$, of an element $\gamma(V) \in \underline{O}_{V}$ with the property that

$$
\gamma\left(V^{\prime}\right) \succeq \underline{O}\left(i_{V^{\prime} V}\right)(\gamma(V))
$$

for all $V^{\prime} \subseteq V$. Clearly every element of $\Gamma \underline{O}$ is a hyper-element, but not conversely.

\footnotetext{
${ }^{13}$ The existence of the $\vee$-operation on $\Gamma \underline{O}$ can be extended to $\underline{O}$ itself. More precisely, there is an arrow $\vee: \underline{O} \times \underline{O} \rightarrow \underline{O}$ where $\underline{O} \times \underline{O}$ denotes the product presheaf over $\mathcal{V}(\mathcal{H})$, whose objects are $(\underline{O} \times \underline{O})_{V}:=\underline{O}_{V} \times \underline{O}_{V}$. Then the arrow $\vee: \underline{O} \times \underline{O} \rightarrow \underline{O}$ is defined at any context $V$ by $\vee_{V}(\hat{\alpha}, \hat{\beta}):=\hat{\alpha} \vee \hat{\beta}$ for all $\hat{\alpha}, \hat{\beta} \in \underline{O}_{V}$.
} 
Now, if $\gamma_{1}$ and $\gamma_{2}$ are hyper-elements, we can define the operations ' $\vee$ ' and ' $\wedge$ ' locally as:

$$
\begin{aligned}
& \left(\gamma_{1} \vee \gamma_{2}\right)(V):=\gamma_{1}(V) \vee \gamma_{2}(V) \\
& \left(\gamma_{1} \wedge \gamma_{2}\right)(V):=\gamma_{1}(V) \wedge \gamma_{2}(V)
\end{aligned}
$$

Because of (2.13) we have, for all $V^{\prime} \subseteq V$,

$$
\begin{aligned}
\underline{O}\left(i_{V^{\prime} V}\right)\left(\left(\gamma_{1} \wedge \gamma_{2}\right)(V)\right) & =\underline{O}\left(i_{V^{\prime} V}\right)\left(\gamma_{1}(V) \wedge \gamma_{2}(V)\right) \\
& \preceq \underline{O}\left(i_{V^{\prime} V}\right)\left(\gamma_{1}(V)\right) \wedge \underline{O}\left(i_{V^{\prime} V}\right)\left(\gamma_{2}(V)\right) \\
& \preceq \gamma_{1}\left(V^{\prime}\right) \wedge \gamma_{2}\left(V^{\prime}\right) \\
& =\left(\gamma_{1} \wedge \gamma_{2}\right)\left(V^{\prime}\right)
\end{aligned}
$$

so that the hyper-element condition (2.16) is preserved.

The occurrence of a logical ' $V$ ' and $\wedge$ ' structure is encouraging, but it is not yet what we want. For one thing, there is no mention of a negation operation; and, anyway, this is not the expected algebra of sub-objects of a 'state space' object. To proceed further we must study more carefully the sub-objects of the spectral presheaf.

\subsection{Daseinisation}

\subsubsection{From Global Sections of $\underline{O}$ to Sub-Objects of $\underline{\Sigma}$.}

The spectral presheaf, $\underline{\Sigma}$, played a central role in the earlier discussions of quantum theory from a topos perspective [13, 14, 15, 16].

Definition 2.3 The spectral presheaf, $\underline{\Sigma}$, is defined as the following functor from $\mathcal{V}(\mathcal{H})^{\text {op }}$ to Sets:

1. On objects $V: \underline{\Sigma}_{V}$ is the Gel'fand spectrum of the unital, abelian subalgebra $V$ of $\mathcal{B}(\mathcal{H})$; i.e., the set of all multiplicative linear functionals $\lambda: V \rightarrow \mathbb{C}$ such that $\lambda(\hat{1})=1$.

2. On morphisms $i_{V^{\prime} V}: V^{\prime} \subseteq V: \underline{\Sigma}\left(i_{V^{\prime} V}\right): \underline{\Sigma}_{V} \rightarrow \underline{\Sigma}_{V^{\prime}}$ is defined by $\underline{\Sigma}\left(i_{V^{\prime} V}\right)(\lambda):=$ $\left.\lambda\right|_{V^{\prime}}$; i.e., the restriction of the functional $\lambda: V \rightarrow \mathbb{C}$ to the subalgebra $V^{\prime} \subseteq V$.

One important result of spectral theory is that $\underline{\Sigma}_{V}$ has a topology that is compact and Hausdorff, and with respect to which the functionals are continuous. This will be important in what follows [7].

The spectral presheaf plays a fundamental role in our research programme as applied to quantum theory. For example, it was shown in the earlier work that the KochenSpecker theorem [9] is equivalent to the statement that $\underline{\Sigma}$ has no global elements. However, $\underline{\Sigma}$ does have sub-objects, and these are central to our scheme: 
Definition 2.4 A sub-object $\underline{S}$ of the spectral presheaf $\underline{\Sigma}$ is a functor $\underline{S}: \mathcal{V}(\mathcal{H})^{o p} \rightarrow$ Sets such that

1. $\underline{S}_{V}$ is a subset of $\underline{\Sigma}_{V}$ for all $V$.

2. If $V^{\prime} \subseteq V$, then $\underline{S}\left(i_{V^{\prime} V}\right): \underline{S}_{V} \rightarrow \underline{S}_{V^{\prime}}$ is just the restriction $\left.\lambda \mapsto \lambda\right|_{V^{\prime}}$ (i.e., the same as for $\underline{\Sigma})$, applied to the elements $\lambda \in \underline{S}_{V} \subseteq \underline{\Sigma}_{V}$.

This definition of a sub-object is standard. However, for our purposes we need something slightly different, namely a 'clopen' sub-object. This is defined to be a subobject $\underline{S}$ of $\underline{\Sigma}$ such that, for all $V$, the set $\underline{S}_{V}$ is a clopen 14 subset of the compact, Hausdorff space $\underline{\Sigma}_{V}$. We denote by $\operatorname{Sub}_{\mathrm{cl}}(\underline{\Sigma})$ the set of all clopen sub-objects of $\underline{\Sigma}$. We will show later that, like $\operatorname{Sub}(\underline{\Sigma})$, the set $\operatorname{Sub}_{\mathrm{cl}}(\underline{\Sigma})$ is a Heyting algebra.

This interest in clopen sets is easy to explain. For, according to the Gel'fand spectral theory, a projection operator $\hat{\alpha} \in \mathcal{P}(V)$ corresponds to a unique clopen subset of the Gelfand spectrum, $\underline{\Sigma}_{V}$. Furthermore, the Gelfand transform $\bar{\alpha}: \underline{\Sigma}_{V} \rightarrow \mathbb{C}$ of $\hat{\alpha}$ takes the values 0,1 only, since the spectrum of a projection operator is just $\{0,1\}$.

It follows that $\bar{\alpha}$ is the characteristic function of the subset, $S_{\hat{\alpha}}$, of $\underline{\Sigma}_{V}$, defined by

$$
S_{\hat{\alpha}}:=\left\{\lambda \in \underline{\Sigma}_{V} \mid \lambda(\hat{\alpha})=1\right\} .
$$

The clopen nature of $S_{\hat{\alpha}}$ follows from the fact that, by the spectral theory, the function $\bar{\alpha}: \underline{\Sigma}_{V} \rightarrow\{0,1\}$ is continuous.

In fact, there is a lattice isomorphism between the lattice $\mathcal{P}(V)$ of projectors in $V$ and the lattice $\mathcal{C} L\left(\underline{\Sigma}_{V}\right)$ of clopen subsets of $\underline{\Sigma}_{V} 15$ given by

$$
\hat{\alpha} \mapsto S_{\hat{\alpha}}:=\left\{\lambda \in \underline{\Sigma}_{V} \mid \lambda(\hat{\alpha})=1\right\} .
$$

Conversely, given a clopen subset $S \in \mathcal{C} L\left(\underline{\Sigma}_{V}\right)$, we get the corresponding projection $\hat{\alpha}$ as the (inverse Gelfand transform of the) characteristic function of $S$. Hence, each $S \in \mathcal{C} L\left(\underline{\Sigma}_{V}\right)$ is of the form $S=S_{\hat{\alpha}}$ for some $\hat{\alpha} \in \mathcal{P}(V)$.

Our claim is the following:

Theorem 2.4 For each projection operator $\hat{P} \in \mathcal{P}(\mathcal{H})$, the collection of subsets

$$
S_{\hat{P}}:=\left\{S_{\delta(\hat{P})_{V}} \subseteq \underline{\Sigma}_{V} \mid V \in \mathrm{Ob}(\mathcal{V}(\mathcal{H}))\right\}
$$

forms a (clopen) sub-object of the spectral presheaf $\underline{\Sigma}$.

Proof. To see this, let $\lambda \in S_{\delta(\hat{P})_{V}}$. Then if $V^{\prime}$ is some abelian subalgebra of $V$, we have $\delta(\hat{P})_{V^{\prime}}=\bigwedge\left\{\hat{Q} \in \mathcal{P}\left(V^{\prime}\right) \mid \hat{Q} \succeq \delta(\hat{P})_{V}\right\} \succeq \delta(\hat{P})_{V}$. Now let $\hat{Q}:=\delta(\hat{P})_{V^{\prime}}-\delta(\hat{P})_{V}$.

\footnotetext{
${ }^{14} \mathrm{~A}$ 'clopen' subset of a topological space is one that is both open and closed.

15 The lattice structure on $\mathcal{C} L\left(\underline{\Sigma}_{V}\right)$ is defined as follows: if $\left(U_{i}\right)_{i \in I}$ is an arbitrary family of clopen subsets of $\underline{\Sigma}_{V}$, then $\bigcup_{i \in I} U_{i}$ is the maximum, and the interior of $\bigcap_{i \in I} U_{i}$ is the minimum of the family. One must take the interior since $\bigcap_{i \in I} U_{i}$ is closed, but not necessarily open.
} 
Then $\lambda\left(\delta(\hat{P})_{V^{\prime}}\right)=\lambda\left(\delta(\hat{P})_{V}\right)+\lambda(\hat{Q})=1$, since $\lambda\left(\delta(\hat{P})_{V}\right)=1$ and $\lambda(\hat{Q}) \in\{0,1\}$. This shows that

$$
\left\{\left.\lambda\right|_{V^{\prime}} \mid \lambda \in S_{\delta(\hat{P})_{V}}\right\} \subseteq S_{\delta(\hat{P})_{V^{\prime}}}
$$

However, the left hand side of (2.26) is the subset $\underline{O}\left(i_{V^{\prime} V}\right)\left(S_{\delta(\hat{P})_{V}}\right) \subseteq \underline{\Sigma}_{V^{\prime}}$ of the outerpresheaf restriction of elements in $S_{\delta(\hat{P})_{V}}$ to $\underline{\Sigma}_{V^{\prime}}$, and the restricted elements all lie in $S_{\delta(\hat{P})_{V^{\prime}}}$. It follows that the collection of sets

$$
S_{\hat{P}}:=\left\{S_{\delta(\hat{P})_{V}} \subseteq \underline{\Sigma}_{V} \mid V \in \mathrm{Ob}(\mathcal{V}(\mathcal{H}))\right\}
$$

forms a (clopen) sub-object of the spectral presheaf $\underline{\Sigma}$.

By these means we have constructed a mapping

$$
\begin{aligned}
\delta: \mathcal{P}(\mathcal{H}) & \longrightarrow \operatorname{Sub}_{\mathrm{cl}}(\underline{\Sigma}) \\
\hat{P} & \mapsto S_{\hat{P}}:=\left\{S_{\delta(\hat{P})_{V}} \mid V \in \mathrm{Ob}(\mathcal{V}(\mathcal{H}))\right\}
\end{aligned}
$$

which sends projection operators on $\mathcal{H}$ to clopen sub-objects of $\underline{\Sigma}$.

\subsubsection{Heidegger encounters physics}

As usual, the projection $\hat{P}$ is regarded as representing a proposition about the quantum system. Thus $\delta$ maps propositions about a quantum system to (clopen) sub-objects of the spectral presheaf. This is remarkably analogous to the situation in classical physics, in which propositions are represented by subsets of the classical state space.

Definition 2.5 The map $\delta$ in (2.28) is a fundamental part of our construction. We call it the daseinisation 16 of $\hat{P}$. We shall use the same word to refer to the operation in eq. 2.1 that relates to the outer presheaf.

We will summarise here some useful properties of daseinisation.

1. The null projection $\hat{0}$ is mapped to the empty sub-object, $\delta(\hat{0})=\left\{\varnothing_{V} \mid V \in\right.$ $\operatorname{Ob}(\mathcal{V}(\mathcal{H}))\}$, of $\underline{\Sigma}$.

2. The identity projection $\hat{1}$ is mapped to the unit sub-object, $\delta(\hat{1})=\left\{\underline{\Sigma}_{V} \mid V \in\right.$ $\operatorname{Ob}(\mathcal{V}(\mathcal{H}))\}=\underline{\Sigma}$ of $\underline{\Sigma}$.

3. Since the daseinisation map $\delta: \mathcal{P}(\mathcal{H}) \rightarrow \Gamma \underline{O}$ is injective (see Section 2.1.2), and the mapping $\Gamma \underline{O} \rightarrow \Gamma\left(P_{\mathrm{cl}} \underline{\Sigma}\right)$ is injective (because there is a monic arrow $\underline{O} \rightarrow P_{\text {cl }} \underline{\Sigma}$ in $\operatorname{Sets}^{\mathcal{V}(\overline{\mathcal{H}})^{\mathrm{op}}}$; see Section 4.3.2), it follows that the daseinisation map $\delta: \mathcal{P}(\mathcal{H}) \rightarrow \Gamma\left(P_{\mathrm{cl}} \underline{\Sigma}\right) \simeq \operatorname{Sub}_{\mathrm{cl}}(\underline{\Sigma})$ is injective. Thus no information about the projector $\hat{P}$ is lost when it is daseinised to become $\delta(\hat{P})$.

\footnotetext{
${ }^{16}$ The expression 'daseinisation' comes from the German word Dasein, which plays a central role in Heidegger's existential philosophy. Dasein translates to 'existence' or, in the very literal sense often stressed by Heidegger, to being-there-in-the-world (the hyphens are very important). Thus daseinisation 'brings-a-quantum-proposition-into-existence' (the hyphens are very important) by hurling it into the classical snap-shots of the world provided by the category of contexts.
} 


\subsection{The Heyting Algebra Structure on $\operatorname{Sub}_{\mathrm{cl}}(\underline{\Sigma})$}

The reason for daseinising projections is that the set, $\operatorname{Sub}(\underline{\Sigma})$, of sub-objects of the spectral presheaf forms a Heyting algebra. Thus the idea is to find a map $\pi_{\mathrm{qt}}: \mathcal{P} \mathcal{L}(S)_{0} \rightarrow$ $\operatorname{Sub}(\underline{\Sigma})$ and then extend it to all of $\mathcal{P} \mathcal{L}(S)$ using the simple recursion ideas discussed in paper I [1].

In our case, the act of daseinisation gives a map from the projection operators to the clopen sub-objects of $\operatorname{Sub}(\underline{\Sigma})$, and therefore a map $\pi_{\mathrm{qt}}: \mathcal{P} \mathcal{L}(S)_{0} \rightarrow \operatorname{Sub}_{\mathrm{cl}}(\underline{\Sigma})$ can be defined by

$$
\pi_{\mathrm{qt}}(A \varepsilon \Delta):=\delta(\hat{E}[A \in \Delta])
$$

However, to extend this definition to $\mathcal{P} \mathcal{L}(S)$, it is necessary to show that the set of clopen sub-objects, $\operatorname{Sub}_{\mathrm{cl}}(\underline{\Sigma})$, is a Heyting algebra. This is not completely obvious from the definition alone.

Theorem 2.5 The collection, $\mathrm{Sub}_{\mathrm{cl}}(\underline{\Sigma})$, of all clopen sub-objects of $\underline{\Sigma}$ is a Heyting algebra.

Proof. First recall how a Heyting algebra structure is placed on the set, $\operatorname{Sub}(\underline{\Sigma})$, of all sub-objects of $\underline{\underline{\Sigma}}$.

The ' $\vee$ '- and ' $\wedge$ '-operations. Let $\underline{S}, \underline{T}$ be two sub-objects of $\underline{\Sigma}$. Then the ' $\vee$ ' and ' $\wedge$ ' operations are defined by

$$
\begin{aligned}
(\underline{S} \vee \underline{T})_{V} & :=\underline{S}_{V} \cup \underline{T}_{V} \\
(\underline{S} \wedge \underline{T})_{V} & :=\underline{S}_{V} \cap \underline{T}_{V}
\end{aligned}
$$

for all contexts $V$. It is easy to see that if $\underline{S}$ and $\underline{T}$ are clopen sub-objects of $\underline{\Sigma}$, then so are $\underline{S} \vee \underline{T}$ and $\underline{S} \wedge \underline{T}$.

The zero and unit elements. The zero element in the Heyting algebra $\mathrm{Sub}(\underline{\Sigma})$ is the empty sub-object $\underline{0}:=\left\{\varnothing_{V} \mid V \in \mathrm{Ob}(\mathcal{V}(\mathcal{H}))\right\}$, where $\varnothing_{V}$ is the empty subset of $\underline{\Sigma}_{V}$. The unit element in $\operatorname{Sub}(\underline{\Sigma})$ is $\underline{\Sigma}$. It is clear that both $\underline{0}$ and $\underline{\Sigma}$ are clopen sub-objects of $\underline{\underline{\Sigma}}$.

The ' $\Rightarrow$ '-operation. The most interesting part is the definition of the implication $\underline{S} \Rightarrow \underline{T}$. For all $V \in \operatorname{Ob}(\mathcal{V}(\mathcal{H}))$, it is given by

$$
\begin{aligned}
(\underline{S} \Rightarrow \underline{T})_{V}:= & \left\{\lambda \in \underline{\Sigma}_{V} \mid \forall V^{\prime} \subseteq V,\right. \text { if } \\
& \left.\underline{\Sigma}\left(i_{V^{\prime} V}\right)(\lambda) \in \underline{S}_{V^{\prime}} \text { then } \underline{\Sigma}\left(i_{V^{\prime} V}\right)(\lambda) \in \underline{T}_{V^{\prime}}\right\} \\
= & \left\{\lambda \in \underline{\Sigma}_{V} \mid \forall V^{\prime} \subseteq V,\right. \text { if } \\
& \left.\left.\lambda\right|_{V^{\prime}} \in \underline{S}_{V^{\prime}} \text { then }\left.\lambda\right|_{V^{\prime}} \in \underline{T}_{V^{\prime}}\right\} .
\end{aligned}
$$


Since $\neg \underline{S}:=\underline{S} \Rightarrow \underline{0}$, the expression for negation follows from the above as

$$
\begin{aligned}
(\neg \underline{S})_{V} & =\left\{\lambda \in \underline{\Sigma}_{V} \mid \forall V^{\prime} \subseteq V, \underline{\Sigma}\left(i_{V^{\prime} V}\right)(\lambda) \notin \underline{S}_{V^{\prime}}\right\} \\
& =\left\{\lambda \in \underline{\Sigma}_{V}\left|\forall V^{\prime} \subseteq V, \lambda\right|_{V^{\prime}} \notin \underline{S}_{V^{\prime}}\right\} .
\end{aligned}
$$

We rewrite the formula for negation as

$$
(\neg \underline{S})_{V}=\bigcap_{V^{\prime} \subseteq V}\left\{\lambda \in \underline{\Sigma}_{V}|\lambda|_{V^{\prime}} \in \underline{S}_{V^{\prime}}^{c}\right\}
$$

where $\underline{S}_{V^{\prime}}{ }^{c}$ denotes the complement of $\underline{S}_{V^{\prime}}$ in $\underline{\Sigma}_{V^{\prime}}$. Clearly, $\underline{S}_{V^{\prime}}{ }^{c}$ is clopen in $\underline{\Sigma}_{V^{\prime}}$ since $\underline{S}_{V^{\prime}}$ is clopen. Since the restriction $\underline{\Sigma}\left(i_{V^{\prime} V}\right): \underline{\Sigma}_{V} \rightarrow \underline{\Sigma}_{V^{\prime}}$ is continuous and surjective 17 , it is easy to see that the inverse image $\underline{\Sigma}\left(i_{V^{\prime} V}\right)^{-1}\left(\underline{S}_{V^{\prime}}^{c}\right)$ is clopen in $\underline{\Sigma}_{V}$. Clearly,

$$
\underline{\Sigma}\left(i_{V^{\prime} V}\right)^{-1}\left(\underline{S}_{V^{\prime}}{ }^{c}\right)=\left\{\lambda \in \underline{\Sigma}_{V}|\lambda|_{V^{\prime}} \in \underline{S}_{V^{\prime}}^{c}\right\}
$$

and so, from (2.36) we have

$$
(\neg \underline{S})_{V}=\bigcap_{V^{\prime} \subseteq V} \underline{\Sigma}\left(i_{V^{\prime} V}\right)^{-1}\left(\underline{S}_{V^{\prime}}{ }^{c}\right)
$$

The problem is that we want $(\neg \underline{S})_{V}$ to be a clopen subset of $\underline{\Sigma}_{V}$. Now the right hand side of (2.38) is the intersection of a family, parameterised by $\left\{V^{\prime} \mid V^{\prime} \subseteq V\right\}$, of clopen sets. Such an intersection is always closed, but it is only guaranteed to be open if $\left\{V^{\prime} \mid V^{\prime} \subseteq V\right\}$ is a finite set, which of course may not be the case.

If $V^{\prime \prime} \subseteq V^{\prime}$ and $\left.\lambda\right|_{V^{\prime \prime}} \in \underline{S}_{V^{\prime \prime}}{ }^{c}$, then $\left.\lambda\right|_{V^{\prime}} \in \underline{S}_{V^{\prime}}{ }^{c}$. Indeed, if we had $\left.\lambda\right|_{V^{\prime}} \in \underline{S}_{V^{\prime}}$, then $\left.\left(\left.\lambda\right|_{V^{\prime}}\right)\right|_{V^{\prime \prime}}=\left.\lambda\right|_{V^{\prime \prime}} \in \underline{S}_{V^{\prime}}$ by the definition of a sub-object, so we would have a contradiction. This implies $\underline{\Sigma}\left(i_{V^{\prime \prime} V}\right)^{-1}\left(\underline{S}_{V^{\prime \prime}}{ }^{c}\right) \subseteq \underline{\Sigma}\left(i_{V^{\prime} V}\right)^{-1}\left(S_{V^{\prime}}{ }^{c}\right)$, and hence the right hand side of (2.38) is a decreasing net of clopen subsets of $\underline{\Sigma}_{V}$ which converges to something, which we take as the subset of $\underline{\Sigma}_{V}$ that is to be $(\neg \underline{S})_{V}$.

Here we have used the fact that the set of clopen subsets of $\underline{\Sigma}_{V}$ is a complete lattice, where the minimum of a family $\left(U_{i}\right)_{i \in I}$ of clopen subsets is defined as the interior of $\bigcap_{i \in I} U_{i}$. This leads us to define

$$
\begin{aligned}
(\neg \underline{S})_{V} & :=\operatorname{int} \bigcap_{V^{\prime} \subseteq V} \underline{\Sigma}\left(i_{V^{\prime} V}\right)^{-1}\left(\underline{S}_{V^{\prime}}^{c}\right) \\
& =\operatorname{int} \bigcap_{V^{\prime} \subseteq V}\left\{\lambda \in \underline{\Sigma}_{V}|\lambda|_{V^{\prime}} \in\left(S_{V^{\prime}}^{c}\right)\right\}
\end{aligned}
$$

as the negation in $\operatorname{Sub}_{\mathrm{cl}}(\underline{\Sigma})$. This modified definition guarantees that $\neg \underline{S}$ is a clopen sub-object. A straightforward extension of this method gives a consistent definition of $\underline{S} \Rightarrow \underline{T}$.

This concludes the proof of the theorem.

\footnotetext{
${ }^{17}$ See proof of Theorem 3.1 below.
} 
The important conclusion of this analysis is that $\operatorname{Sub}_{\mathrm{cl}}(\underline{\underline{\Sigma}})$ is a Heyting algebra. It is the subset of $\operatorname{Sub}(\underline{\Sigma})$ that incorporates the spectral topology on the stalks $\underline{\Sigma}_{V}$, and the spectral-theory association of projection operators in $V$ with clopen subsets of $\underline{\Sigma}_{V}$. In particular, the map $\pi_{\mathrm{qt}}: \mathcal{P} \mathcal{L}(S)_{0} \rightarrow \operatorname{Sub}_{\mathrm{cl}}(\underline{\Sigma})$ given by

$$
\pi_{\mathrm{qt}}(A \varepsilon \Delta):=\delta(\hat{E}[A \in \Delta])
$$

can now be extended to all of $\mathcal{P} \mathcal{L}(S)$ using the ideas discussed in the first paper.

In conclusion: daseinisation can be used to give a representation/model of the language $\mathcal{P} \mathcal{L}(S)$ in the Heyting algebra $\operatorname{Sub}_{\mathrm{cl}}(\underline{\underline{\Sigma}})$.

\subsection{Daseinisation and the Operations of Quantum Logic.}

It is interesting to ask to what extent, if any, the map $\delta: \mathcal{P}(\mathcal{H}) \rightarrow \operatorname{Sub}_{\mathrm{cl}}(\underline{\Sigma})$ respects the lattice structure on $\mathcal{P}(\mathcal{H})$. Of course, we know that it cannot be completely preserved since the quantum logic $\mathcal{P}(\mathcal{H})$ is non-distributive, whereas $\operatorname{Sub}_{\mathrm{cl}}(\underline{\Sigma})$ is a Heyting algebra, and hence distributive.

We saw in Section 2.1.3 that, for the mapping $\delta: \mathcal{P}(\mathcal{H}) \rightarrow \Gamma \underline{O}$, we have

$$
\begin{aligned}
& \delta(\hat{P} \vee \hat{Q})_{V}=\delta(\hat{P})_{V} \vee \delta(\hat{Q})_{V} \\
& \delta(\hat{P} \wedge \hat{Q})_{V} \preceq \delta(\hat{P})_{V} \wedge \delta(\hat{Q})_{V}
\end{aligned}
$$

for all contexts $V$ in $\mathrm{Ob}(\mathcal{V}(\mathcal{H}))$.

The clopen subset of $\underline{\Sigma}_{V}$ that corresponds to $\delta(\hat{P})_{V} \vee \delta(\hat{Q})_{V}$ is $S_{\delta(\hat{P})_{V}} \cup S_{\delta(\hat{Q})_{V}}$. This implies that the daseinisation map $\delta: \mathcal{P}(\mathcal{H}) \rightarrow \operatorname{Sub}_{\mathrm{cl}}(\underline{\Sigma})$ is a morphism of $\vee$-semilattices.

On the other hand, $\delta(\hat{P})_{V} \wedge \delta(\hat{Q})_{V}$ corresponds to the subset $S_{\delta(\hat{P})_{V}} \cap S_{\delta(\hat{Q})_{V}}$ of $\underline{\Sigma}_{V}$. Therefore, since $S_{\delta(\hat{P} \wedge \hat{Q})_{V}} \subseteq S_{\delta(\hat{P})_{V}} \cap S_{\delta(\hat{Q})_{V}}$, daseinisation is not a morphism of $\wedge$-semi-lattices. In summary, for all projectors $\hat{P}, \hat{Q}$ we have

$$
\begin{aligned}
& \delta(\hat{P} \vee \hat{Q})=\delta(\hat{P}) \vee \delta(\hat{Q}) \\
& \delta(\hat{P} \wedge \hat{Q}) \preceq \delta(\hat{P}) \wedge \delta(\hat{Q})
\end{aligned}
$$

where the logical connectives on the left hand side lie in the quantum $\operatorname{logic} \mathcal{P}(\mathcal{H})$, and those on the right hand side lie in the Heyting algebra $\operatorname{Sub}_{\mathrm{cl}}(\underline{\Sigma})$, as do the symbols '=' and ' $\preceq$ '.

As remarked above, it is not surprising that (2.45) is not an equality. Indeed, the quantum logic $\mathcal{P}(\mathcal{H})$ is non-distributive, whereas the Heyting algebra $\operatorname{Sub}_{\mathrm{cl}}(\underline{\Sigma})$ is distributive, and so it would be impossible for both (2.44) and (2.45) to be equalities. The inequality in (2.45) is the price that must be paid for liberating the projection operators from the shackles of quantum logic and thrusting them down to the existential world of Heyting algebras. 


\subsubsection{The Status of the Possible Axiom ' $A \varepsilon \Delta_{1} \wedge A \varepsilon \Delta_{2} \Leftrightarrow A \varepsilon \Delta_{1} \cap \Delta_{2}$ '}

We have the representation in (2.29), $\pi_{\mathrm{qt}}(A \varepsilon \Delta):=\delta(\hat{E}[A \in \Delta])$, of the primitive propositions $A \varepsilon \Delta$, and, as explained in our first paper, [1], this can be extended to compound sentences by making the obvious definitions:

$$
\begin{array}{ll}
\text { (a) } & \pi_{\mathrm{qt}}(\alpha \vee \beta):=\pi_{\mathrm{qt}}(\alpha) \vee \pi_{\mathrm{qt}}(\beta) \\
(b) & \left.\pi_{\mathrm{qt}}(\alpha \wedge \beta):=\pi_{\mathrm{qt}}(\alpha) \wedge \pi_{\mathrm{qt}} \beta\right) \\
(c) & \pi_{\mathrm{qt}}(\neg \alpha):=\neg \pi_{\mathrm{qt}}(\alpha) \\
\text { (d) } & \pi_{\mathrm{qt}}(\alpha \Rightarrow \beta):=\pi_{\mathrm{qt}}(\alpha) \Rightarrow \pi_{\mathrm{qt}}(\beta)
\end{array}
$$

As a result, we necessarily get a representation of the full language $\mathcal{P} \mathcal{L}(S)$ in the Heyting algebra $\operatorname{Sub}_{\mathrm{cl}}(\underline{\Sigma})$. However, we then find that:

$$
\begin{aligned}
\pi_{\mathrm{qt}}\left(A \varepsilon \Delta_{1} \wedge A \varepsilon \Delta_{2}\right) & :=\pi_{\mathrm{qt}}\left(A \varepsilon \Delta_{1}\right) \wedge \pi_{\mathrm{qt}}\left(A \varepsilon \Delta_{2}\right) \\
& =\delta\left(\hat{E}\left[A \in \Delta_{1}\right]\right) \wedge \delta\left(\hat{E}\left[A \in \Delta_{2}\right]\right) \\
& \succeq \delta\left(\hat{E}\left[A \in \Delta_{1}\right] \wedge \hat{E}\left[A \in \Delta_{2}\right]\right) \\
& \left.=\delta\left(\hat{E}\left[A \in \Delta_{1} \cap \Delta_{2}\right)\right]\right) \\
& =\pi_{\mathrm{qt}}\left(A \varepsilon \Delta_{1} \cap \Delta_{2}\right)
\end{aligned}
$$

where, (2.52) comes from (2.45), and in (2.53) we have used the property of spectral projectors that $\left.\hat{E}\left[A \in \Delta_{1}\right] \wedge \hat{E}\left[A \in \Delta_{2}\right]=\hat{E}\left[A \in \Delta_{1} \cap \Delta_{2}\right)\right]$. Thus, although by definition, $\pi_{\mathrm{qt}}\left(A \varepsilon \Delta_{1} \wedge A \varepsilon \Delta_{2}\right)=\pi_{\mathrm{qt}}\left(A \varepsilon \Delta_{1}\right) \wedge \pi_{\mathrm{qt}}\left(A \varepsilon \Delta_{2}\right)$, we only have the inequality

$$
\pi_{\mathrm{qt}}\left(A \varepsilon \Delta_{1} \cap \Delta_{2}\right) \preceq \pi_{\mathrm{qt}}\left(A \varepsilon \Delta_{1} \wedge A \varepsilon \Delta_{2}\right)
$$

On the other hand, the same line of argument shows that

$$
\pi_{\mathrm{qt}}\left(A \varepsilon \Delta_{1} \vee A \varepsilon \Delta_{2}\right)=\pi_{\mathrm{qt}}\left(A \varepsilon \Delta_{1} \cup \Delta_{2}\right)
$$

Thus we could consistently add the axiom

$$
A \varepsilon \Delta_{1} \vee A \varepsilon \Delta_{2} \Leftrightarrow A \varepsilon \Delta_{1} \cup \Delta_{2}
$$

to the language $\mathcal{P} \mathcal{L}(S)$, but not

$$
A \varepsilon \Delta_{1} \wedge A \varepsilon \Delta_{2} \Leftrightarrow A \varepsilon \Delta_{1} \cap \Delta_{2}
$$

Of, course, both axioms are consistent with the representation of $\mathcal{P} \mathcal{L}(S)$ in classical physics.

It should be emphasised that there is nothing wrong with this result: indeed, as stated above, it is the necessary price to be paid for forcing a non-distributive algebra to have a 'representation' in a Heyting algebra. 


\subsubsection{Inner Daseinisation and $\delta(\neg \hat{P})$.}

In the same spirit, one might ask about " $\neg(A \varepsilon \Delta)$ ". By definition, as in (2.49), we have $\pi_{\mathrm{qt}}(\neg(A \varepsilon \Delta)):=\neg \pi_{\mathrm{qt}}(A \varepsilon \Delta)=\neg \delta(\hat{E}[A \in \Delta])$. However, the question then is how, if at all, this is related to $\delta(\hat{E}[A \in \mathbb{R} / \Delta])$, bearing in mind the axiom

$$
\neg(A \varepsilon \Delta) \Leftrightarrow A \varepsilon \mathbb{R} \backslash \Delta
$$

that can be added to the classical representation of $\mathcal{P} \mathcal{L}(S)$. Thus something needs to be said about $\delta(\neg \hat{P})$, where $\neg \hat{P}=\hat{1}-\hat{P}$ is the negation operation in the quantum $\operatorname{logic} \mathcal{P}(\mathcal{H})$.

In order to express $\delta(\neg P)$ in terms of $\delta(\hat{P})$, we need to introduce another operation:

Definition 2.6 The inner daseinisation, $\delta^{i}(\hat{P})$, of $\hat{P}$ is defined for each context $V$ as

$$
\delta^{i}(\hat{P})_{V}:=\bigvee\{\hat{Q} \in \mathcal{P}(V) \mid \hat{Q} \preceq \hat{P}\} .
$$

This should be contrasted with the definition of outer daseinisation in eq. (2.1).

Thus $\delta^{i}(\hat{P})_{V}$ is the best approximation that can be made to $\hat{P}$ by taking the 'largest' projector in $V$ that implies $\hat{P}$.

As with the other daseinisation construction, this operation was first introduced by de Groote in [10] where he called it the core of the projection operator $\hat{P}$. We prefer to use the phrase 'inner daseinisation', and then to refer to (2.1) as the 'outer daseinisation' operation on $\hat{P}$. The existing notation $\delta(\hat{P})_{V}$ will be replaced with $\delta^{o}(\hat{P})_{V}$ if there is any danger of confusing the two daseinisation operations.

With the aid of inner daseinisation, a new presheaf, $\underline{I}$, can be constructed as an exact analogue of the the outer presheaf, $\underline{O}$, defined in Section 2.1.1. Specifically:

Definition 2.7 The inner presheaf $\underline{I}$ is defined over the category $\mathcal{V}(\mathcal{H})$ as follows:

(i) On objects $V \in \operatorname{Ob}(\mathcal{V}(\mathcal{H})):$ We define $\underline{I}_{V}:=\mathcal{P}(V)$

(ii) On morphisms $i_{V^{\prime} V}: V^{\prime} \subseteq V:$ The mapping $\underline{I}\left(i_{V^{\prime} V}\right): \underline{I}_{V} \rightarrow \underline{I}_{V^{\prime}}$ is defined as $\underline{I}\left(i_{V^{\prime} V}\right)(\hat{\alpha}):=\delta^{i}(\hat{\alpha})_{V}$ for all $\hat{\alpha} \in \mathcal{P}(V)$.

It is easy to see that the collection $\left\{\delta^{i}(\hat{P})_{V} \mid V \in \mathrm{Ob}(\mathcal{V}(\mathcal{H}))\right\}$ of projection operators given by (2.60) is a global element of $\underline{I}$.

It is also straightforward to show that

$$
\underline{O}\left(i_{V^{\prime} V}\right)(\neg \hat{\alpha})=\neg \underline{I}\left(i_{V^{\prime} V}\right)(\hat{\alpha})
$$

for all projectors $\hat{\alpha}$ in $V$, and for all $V^{\prime} \subseteq V$. It follows from (2.61) that

$$
\delta^{o}(\neg \hat{P})_{V}=\hat{1}-\delta^{i}(\hat{P})_{V}
$$


for all projectors $\hat{P}$ and all contexts $V$.

It is clear from (2.61) that the negation operation on projectors defines a map $\neg$ : $\Gamma \underline{O} \rightarrow \Gamma \underline{I}, \gamma \mapsto \neg \gamma$; i.e., for all contexts $V$, we map $\gamma(V) \mapsto \neg \gamma(V):=\hat{1}-\gamma(V)$. In fact, one can go further than this and show that there is a natural transformation between the two presheaves $\underline{O}$ and $\underline{I}$ which is an isomorphism in the category $\operatorname{Sets}^{\mathcal{V}(\mathcal{H})^{\text {op }}}$; i.e., $\underline{O}$ and $\underline{I}$ are isomorphic objects in the topos. This isomorphism means that, in principle, we can always work with one presheaf only. We have elected to use the outer presheaf $\underline{O}$ and, as a result, only the outer daseinisation (which we call just 'daseinisation') operation.

\section{The Special Nature of Daseinised Projections}

\subsection{Daseinised Projections as Optimal Sub-Objects}

We have shown how daseinisation leads to an interpretation/model of the language $\mathcal{P} \mathcal{L}(S)$ in the Heyting algebra $\operatorname{Sub}_{\mathrm{cl}}(\underline{\Sigma})$. The primitive propositions " $A \varepsilon \Delta$ ", i.e., the elements of $\mathcal{P} \mathcal{L}(S)_{0}$, are represented by the clopen sub-objects $\delta(\hat{P})$, where $\hat{P}=\hat{E}[A \in \Delta]$ is the spectral projection of $\hat{A}$ that corresponds to the Borel set $\Delta$.

We also saw that, in general, the 'minimum' $\delta(\hat{P}) \wedge \delta(\hat{Q})$ of two projection operators $\hat{P}$ and $\hat{Q}$, is not itself of the form $\delta(\hat{R})$ for any projector $\hat{R}$. The same applies to the negation $\neg \delta(\hat{P})$.

This raises the question of whether the sub-objects of $\underline{\Sigma}$ that are of the form $\delta(\hat{P})$ can be characterised in a simple way. Rather interestingly, the answer is 'yes' as we will now see.

Let $V^{\prime}, V \in \mathrm{Ob}(\mathcal{V}(\mathcal{H}))$ be such that $V^{\prime} \subseteq V$. As one might expect, there is a close connection between the restriction $\underline{O}\left(i_{V^{\prime} V}\right): \underline{O}_{V} \rightarrow \underline{O}_{V^{\prime}}, \delta(\hat{P})_{V} \mapsto \delta(\hat{P})_{V^{\prime}}$, of the outer presheaf, and the restriction $\underline{\Sigma}\left(i_{V^{\prime} V}\right): \underline{\Sigma}_{V} \rightarrow \underline{\Sigma}_{V^{\prime}},\left.\lambda \mapsto \lambda\right|_{V^{\prime}}$, of the spectral presheaf. Indeed, if $\hat{P} \in \mathcal{P}(\mathcal{H})$ is a projection, and $S_{\delta(\hat{P})_{V}} \subseteq \underline{\Sigma}_{V}$ is defined as in (2.23), we have the following result:

\section{Theorem 3.1}

$$
S_{\underline{Q}\left(i_{V^{\prime} V}\right)\left(\delta(\hat{P})_{V}\right)}=\underline{\Sigma}\left(i_{V^{\prime} V}\right)\left(S_{\delta(\hat{P})_{V}}\right) .
$$

Before proving the theorem, we need a few preparations. For typographical simplicity, for a given pair $V^{\prime} \subseteq V$ we define the map

$$
\begin{aligned}
r: P\left(\underline{\Sigma}_{V}\right) & \rightarrow P\left(\underline{\Sigma}_{V^{\prime}}\right) \\
S & \mapsto \underline{\Sigma}\left(i_{V^{\prime} V}\right)(S)=\left\{\left.\lambda\right|_{V^{\prime}} \mid \lambda \in S\right\},
\end{aligned}
$$

where $P\left(\underline{\Sigma}_{V}\right)$ is the power set of $\underline{\Sigma}_{V}$. We then have

Lemma 3.2 If $\delta(\hat{P})_{V^{\prime}}=\delta(\hat{P})_{V}$, then every $\lambda_{V^{\prime}} \in S_{\delta(\hat{P})_{V^{\prime}}}$ is of the form $\left.\lambda_{V}\right|_{V^{\prime}}$ for some $\lambda_{V} \in S_{\delta(\hat{P})_{V}}$. This implies $r\left(S_{\delta(\hat{P})_{V}}\right)=S_{\delta(\hat{P})_{V^{\prime}}}$. 
Proof. If $\lambda_{V} \in S_{\delta(\hat{P})_{V}}$, then $\left.\lambda_{V}\right|_{V^{\prime}} \in S_{\delta(\hat{P})_{V^{\prime}}}\left(\right.$ since $\left.\left.\lambda_{V}\right|_{V^{\prime}}\left(\delta(\hat{P})_{V^{\prime}}\right)=\left.\lambda_{V}\right|_{V^{\prime}}\left(\delta(\hat{P})_{V}\right)=1\right)$, whereas if $\lambda_{V} \notin S_{\delta(\hat{P})_{V}}$, then $\left.\lambda_{V}\right|_{V^{\prime}} \notin S_{\delta(\hat{P})_{V^{\prime}}}\left(\right.$ since $\left.\left.\lambda_{V}\right|_{V^{\prime}}\left(\delta(\hat{P})_{V^{\prime}}\right)=\left.\lambda_{V}\right|_{V^{\prime}}\left(\delta(\hat{P})_{V}\right)=0\right)$. Since every $\lambda_{V^{\prime}} \in \underline{\Sigma}_{V^{\prime}}$ is of the form $\left.\lambda_{V}\right|_{V^{\prime}}=r\left(\lambda_{V}\right)$ for some $\lambda_{V} \in S_{\delta(\hat{P})_{V}}$, the mapping $r: P\left(\underline{\Sigma}_{V}\right) \rightarrow P\left(\underline{\Sigma}_{V^{\prime}}\right)$ sends $S_{\delta(\hat{P})_{V}}$ to $S_{\delta(\hat{P})_{V^{\prime}}}$, and the complement $\left(S_{\delta(\hat{P})_{V}}\right)^{c}$ in $\underline{\Sigma}_{V}$ is sent to the complement $\left(S_{\delta(\hat{P})_{V^{\prime}}}\right)^{c}$ in $\underline{\Sigma}_{V^{\prime}}$.

Proof. (of Theorem 3.1) By definition, $\underline{Q}\left(i_{V^{\prime} V}\right)\left(\delta(\hat{P})_{V}\right)=\delta(\hat{P})_{V^{\prime}}$. The mapping $r$ defined in (3.2) is open and continuous (which follows from Prop. 3.22 in [11] $]^{18}$ ).

The map $r$ is also closed. To see this, let $C \subseteq \underline{\Sigma}_{V}$ be a closed subset. Since $\underline{\Sigma}_{V}$ is compact, $C$ is compact, and since $r$ is continuous, $r(C) \subseteq \Sigma_{V^{\prime}}$ is compact, too. However, $\underline{\Sigma}_{V^{\prime}}$ is Hausdorff, and so $r(C)$ is closed in $\underline{\Sigma}_{V^{\prime}}$. we have

Now, $S_{\delta(\hat{P})_{V}}$ is a clopen subset of $\underline{\Sigma}_{V}$, so $r\left(S_{\delta(\hat{P})_{V}}\right)$ is a clopen subset of $\underline{\Sigma}_{V^{\prime}}$. Clearly,

$$
\begin{aligned}
r\left(S_{\delta(\hat{P})_{V}}\right) & =\operatorname{int} \bigcap\left\{S \subseteq \underline{\Sigma}\left(V^{\prime}\right) \mid S \in \mathcal{C} L\left(\underline{\Sigma}\left(V^{\prime}\right)\right), r\left(S_{\delta(\hat{P})_{V}}\right) \subseteq S\right\} \\
& =\operatorname{int} \bigcap\left\{S_{\hat{Q}} \in \mathcal{C} L\left(\underline{\Sigma}_{V^{\prime}}\right) \mid r\left(S_{\delta(\hat{P})_{V}}\right) \subseteq S_{\hat{Q}}\right\} .
\end{aligned}
$$

We now show that $r\left(S_{\delta(\hat{P})_{V}}\right) \subseteq S_{\hat{Q}}$ implies $\hat{Q} \succeq \delta(\hat{P})_{V}$. Assume that $\hat{Q} \prec \delta(\hat{P})_{V}$. Let $\hat{R}:=\delta(\hat{P})_{V}-\hat{Q} \in \mathcal{P}(V)$, and let $\lambda \in S_{\hat{R}} \subseteq \underline{\Sigma}_{V}$. Then $\lambda \in S_{\delta(\hat{P})_{V}}$, but $\lambda \notin S_{\hat{Q}} \subseteq \Sigma_{V}$. Lemma 3.2 shows that $r\left(S_{\hat{Q}}\right)=S_{\hat{Q}}$ (where the former $S_{\hat{Q}}$ is a subset of $\underline{\Sigma}_{V}$ and the latter is a subset of $\left.\underline{\Sigma}_{V^{\prime}}\right)$ and $r\left(\left(S_{\hat{Q}}\right)^{c}\right)=\left(S_{\hat{Q}}\right)^{c}$. In particular, $\left.\lambda\right|_{V^{\prime}} \notin S_{\hat{Q}} \subseteq \underline{\Sigma}_{V^{\prime}}$, but $\left.\lambda\right|_{V^{\prime}} \in r\left(S_{\delta(\hat{P})_{V}}\right)$, so

$$
\hat{Q} \prec \delta(\hat{P})_{V} \quad \Rightarrow \quad r\left(S_{\delta(\hat{P})_{V}}\right) \nsubseteq S_{\hat{Q}} .
$$

Hence we must have $\hat{Q} \succeq \delta(\hat{P})_{V}$, from which we obtain

$$
r\left(S_{\delta(\hat{P})_{V}}\right)=\operatorname{int} \bigcap\left\{S_{\hat{Q}} \in \mathcal{C} L\left(\underline{\Sigma}_{V^{\prime}}\right) \mid \hat{Q} \succeq \delta(\hat{P})_{V}\right\} .
$$

We now use the lattice isomorphism between $\mathcal{C} L\left(\underline{\Sigma}_{V^{\prime}}\right)$ and $\mathcal{P}\left(V^{\prime}\right)$ (see (2.24 $)$ ) to get

$$
\begin{aligned}
r\left(S_{\delta(\hat{P})_{V}}\right) & =\operatorname{int} \bigcap\left\{S_{\hat{Q}} \in \mathcal{C} \mathcal{L}\left(\underline{\Sigma}_{V^{\prime}}\right) \mid \hat{Q} \succeq \delta(\hat{P})_{V}\right\} \\
& =S_{\left\{\hat{Q} \in \mathcal{P}\left(V^{\prime}\right) \mid \hat{Q} \succeq \delta(\hat{P})_{V}\right\}}=S_{\underline{Q}\left(i_{V^{\prime} V}\right)\left(\delta(\hat{P})_{V}\right)} .
\end{aligned}
$$

This completes the proof of the theorem.

This result shows that the sub-objects $\delta(\hat{P})=\left\{S_{\delta(\hat{P})_{V}} \mid V \in \mathrm{Ob}(\mathcal{V}(\mathcal{H}))\right\}$ of $\underline{\Sigma}$ are of a very special kind. Namely, they are such that the restriction

$$
\underline{\Sigma}\left(i_{V^{\prime} V}\right): S_{\delta(\hat{P})_{V}} \rightarrow S_{\delta(\hat{P})_{V^{\prime}}}
$$

\footnotetext{
${ }^{18}$ The von Neumann algebra $V$ corresponds to the algebra $\mathcal{R}$ in de Groote's (more general) proof, $V^{\prime}$ corresponds to $\mathcal{A}$. Our mapping $r$ is his $\zeta_{\mathcal{A}}$. For abelian von Neumann algebras, quasipoints can be identified with elements of the Gel'fand spectrum, and the topologies on the space $\mathcal{Q}(V)$ of quasipoints and the Gel'fand spectrum $\underline{\Sigma}_{V}$ coincide. For all this, see [11, in particular, Thm. 3.2.
} 
is a surjective mapping of sets.

For an arbitrary sub-object $\underline{K}$ of $\underline{\Sigma}$, this will generally not be the case and $\underline{\Sigma}\left(i_{V^{\prime} V}\right)$ will only map $\underline{K}_{V}$ into $\underline{K}_{V^{\prime}}$. Indeed, this is essentially the definition of a sub-object of a presheaf. Thus we see that the daseinised projections $S_{\delta(\hat{P})}=\left\{S_{\delta(\hat{P})_{V}} \mid V \in \mathrm{Ob}(\mathcal{V}(\mathcal{H}))\right\}$ are optimal in the following sense. As we go 'down the line' to smaller and smaller subalgebras of a context $V$-for example, from $V$ to $V^{\prime} \subseteq V$, then to $V^{\prime \prime} \subseteq V^{\prime}$ etc.then the subsets $S_{\delta(\hat{P})_{V^{\prime}}}, S_{\delta(\hat{P})_{V^{\prime \prime}}}, \ldots$ are as small as they can be; i.e., $S_{\delta(\hat{P})_{V^{\prime}}}$ is the smallest subset of $\underline{\Sigma}_{V^{\prime}}$ such that $\underline{\Sigma}\left(i_{V^{\prime} V}\right)\left(S_{\delta(\hat{P})_{V}}\right) \subseteq S_{\delta(\hat{P})_{V^{\prime}}}$, likewise $S_{\delta(\hat{P})_{V^{\prime \prime}}}$ is the smallest subset of $\underline{\Sigma}_{V^{\prime \prime}}$ such that $\underline{\Sigma}\left(i_{V^{\prime \prime} V^{\prime}}\right)\left(S_{\delta(\hat{P})_{V^{\prime}}}\right) \subseteq S_{\delta(\hat{P})_{V^{\prime \prime}}}$, and so on.

It is also clear from this result that there are sub-objects of $\underline{\Sigma}$ that are not of the form $\delta(\hat{P})$ for any projector $\hat{P} \in \mathcal{P}(\mathcal{H})$.

These more general sub-objects of $\underline{\Sigma}$ show up explicitly in the representation of the more sophisticated language $\mathcal{L}(S)$. This subject will be discussed in the next paper, III, in this series. There we analyse the representation, $\phi$, of the language $\mathcal{L}(S)$ in the topos $\operatorname{Sets}^{\mathcal{V}(\mathcal{H})^{\text {op }}}$. This involves constructing the quantity-value object $\mathcal{R}_{\phi}$ (to be denoted $\underline{\mathcal{R}}$ ), and then finding the representation of a function symbol $A: \Sigma \rightarrow \mathcal{R}$, from $\mathcal{L}(S)$, in the form of a specific arrow $\breve{A}: \underline{\Sigma} \rightarrow \underline{\mathcal{R}}$ in the topos. The generic sub-objects of $\underline{\Sigma}$ are then of the form $\breve{A}^{-1}(\underline{\Xi})$ for sub-objects $\Xi$ of $\underline{\mathcal{R}}$. This is an illuminating way of studying the sub-objects of $\underline{\Sigma}$ that do not come from the propositional language $\mathcal{P} \mathcal{L}(S)$.

\section{Truth Values in Topos Physics}

\subsection{The Mathematical Proposition " $x \in K$ "}

So far we have concentrated on finding a Heyting-algebra representation of the propositions in quantum theory, but of course there is more to physics than that. We also want to know if a certain proposition is true: a question which, in physical theories, can only be answered by specifying a state of the system, or something that can play an analogous role.

In classical physics, the situation is straightforward. There, a proposition " $A \varepsilon \Delta$ " is represented by the subset $19 \pi_{\mathrm{cl}}(A \varepsilon \Delta):=\breve{A}^{-1}(\Delta) \subseteq \mathcal{S}$ of the state space $\mathcal{S}$; and then, the proposition is true in a state $s$ if and only if $s \in \breve{A}^{-1}(\Delta)$; i.e., if and only if the (micro-) state $s$ belongs to the subset, $\pi_{\mathrm{cl}}(A \varepsilon \Delta)$, of $\mathcal{S}$ that represents the proposition.

Thus, each state $s$ assigns to any primitive proposition " $A \varepsilon \Delta$ ", a truth value, $\nu(A \varepsilon \Delta ; s)$, which lies in the set \{false, true\} (which we identify with $\{0,1\}$ ) and is defined as

$$
\nu(A \varepsilon \Delta ; s):= \begin{cases}1 & \text { if } s \in \pi_{\mathrm{cl}}(A \varepsilon \Delta):=\breve{A}^{-1}(\Delta) \\ 0 & \text { otherwise. }\end{cases}
$$

\footnotetext{
${ }^{19}$ Here, $\breve{A}: \mathcal{S} \rightarrow \mathbb{R}$ is the mathematical representation of the physical quantity $A$.
} 
for all $s \in \mathcal{S}$.

However, the situation is very different for quantum theory. This is because the spectral presheaf $\underline{\Sigma}$, which is the analogue of the classical state space $\mathcal{S}$, has no global elements at all. And no doubt there will be other topos theories of physics where $\Gamma \Sigma_{\phi}$ is empty; or, if $\Sigma_{\phi}$ does have global elements, there are not enough of them to determine $\Sigma_{\phi}$ as an object in the topos. In this circumstance, a new concept is required to replace the familiar idea of a 'state of the system'.

In physics, the propositions of interest are of the form " $A \varepsilon \Delta$ ", which refer to the value of a physical quantity. However, in constructing a theory of physics, physical propositions are invariably first translated into mathematical propositions; the concept of 'truth' is then studied in the context of the latter.

Let us start with set-theory based mathematics, where the most basic proposition is of the form " $x \in K$ ", where $K$ is a subset of a set $X$, and $x$ is an element of $X$. Then the truth value, denoted $\nu(x \in K)$, of the proposition " $x \in K$ " is

$$
\nu(x \in K)= \begin{cases}1 & \text { if } x \text { belongs to } K \\ 0 & \text { otherwise. }\end{cases}
$$

Thus the proposition " $x \in K$ " is true if, and only if, $x$ belongs to $K$; i.e., if, and only if, $x \in K$.

This result sounds like a tautology but, nevertheless, it is the foundation of the assignment of truth values in physics. For example, in classical physics, if the state is $s$, the truth value, $\nu(A \varepsilon \Delta ; s)$, of the physical proposition " $A \varepsilon \Delta$ " is defined to be the truth value of the mathematical proposition " $\breve{A}(s) \in \Delta$ "; or, equivalently, of the mathematical proposition " $s \in \breve{A}^{-1}(\Delta)$ ".

Thus, using (4.2), we get, for all $s \in \mathcal{S}$,

$$
\nu(A \varepsilon \Delta ; s):= \begin{cases}1 & \text { if } s \in \breve{A}^{-1}(\Delta) ; \\ 0 & \text { otherwise. }\end{cases}
$$

which reproduces (4.1). We now consider the analogue of the above in a general topos $\tau$.

Let $X$ be an object in the topos $\tau$, and let $K$ be a sub-object of $X$. Then $K$ is determined by a characteristic arrow $\chi_{K}: X \rightarrow \Omega_{\tau}$, where $\Omega_{\tau}$ is the sub-object classifier for the topos; equivalently we have an arrow $\ulcorner K\urcorner: 1_{\tau} \rightarrow P X$.

Now suppose that $20\ulcorner x\urcorner: 1_{\tau} \rightarrow X$ is a global element of $X$; i.e., $x \in \Gamma X$. Then the truth value of the mathematical proposition " $x \in K$ " is defined to be

$$
\nu(x \in K):=\chi_{K} \circ\ulcorner x\urcorner
$$

\footnotetext{
${ }^{20}$ One of the basic properties of a topos is that there is a one-to-one correspondence between arrows $f: A \times B \rightarrow \Omega$ and arrows $\ulcorner f\urcorner: A \rightarrow P B:=\Omega^{B}$. In general, $\ulcorner f\urcorner$ is called the power transpose of $f$. If $A \simeq 1$ then $\ulcorner f\urcorner$ is known as the name of the arrow $f: B \rightarrow \Omega$. On exponentials, see the Appendix in paper I.
} 
where $\chi_{K} \circ\ulcorner x\urcorner: 1_{\tau} \rightarrow \Omega_{\tau}$. Thus $\nu(x \in K)$ is an element of $\Gamma \Omega_{\tau}$; i.e., it is a global element of the sub-object classifier $\Omega_{\tau}$.

The relation with the result (4.2) (in the topos Sets) can be seen by noting that, in (4.2), the characteristic function of the subset $K \subseteq X$ is the function $\chi_{K}: X \rightarrow\{0,1\}$ such that $\chi_{K}(x)=1$ if $x \in K$, and $\chi_{K}(x)=0$ otherwise. It follows that (4.2) can be rewritten as

$$
\begin{aligned}
\nu(x \in K) & =\chi_{K}(x) \\
& =\chi_{K} \circ\ulcorner x\urcorner
\end{aligned}
$$

where, in (4.5), $\ulcorner x\urcorner$ is the function $\ulcorner x\urcorner:\{*\} \rightarrow X$ that is defined by $\ulcorner x\urcorner(*):=x$. The link with (4.4) is clear when one remembers that, in the topos Sets, the terminal object, $1_{\text {Sets }}$, is just the singleton set $\{*\}$.

In quantum theory, the topos is $\operatorname{Sets}^{\mathcal{V}(\mathcal{H})^{\mathrm{op}}}$, and so the objects are all presheaves. In particular, at each stage $V$, the sub-object classifier $\underline{\Omega}:=\Omega_{\operatorname{Sets}(\mathcal{H})^{\text {op }}}$ is the set of sieves on $V$. In this case, if $\underline{K}$ is a sub-object of $\underline{X}$, and $x \in \Gamma \underline{X}$, the explicit form for (4.5) is the sieve

$$
\nu(x \in \underline{K})_{V}:=\left\{V^{\prime} \subseteq V \mid x_{V^{\prime}} \in \underline{K}_{V^{\prime}}\right\}
$$

at each stage $V \in \mathrm{Ob}(\mathcal{V}(\mathcal{H}))$.

The definitions (4.4) and (4.6) play a central role in constructing truth values in our scheme. However, as $\underline{\Sigma}$ has no global elements, these truth values cannot be derived from an expression $\nu(s \in \underline{K})$ with $\ulcorner s\urcorner: 1_{\operatorname{Sets} \mathcal{\nu}(\mathcal{H})^{\text {op }}} \rightarrow \underline{\Sigma}$. Therefore, we must proceed in a different way, as will become clear by the end of the following Section.

\subsection{Truth Objects}

\subsubsection{Linguistic Aspects of Truth Objects.}

To understand the construction of 'truth values' in a topos we return again to the discussion in paper I of local languages [1. In this Section we will employ the local language $\mathcal{L}(S)$, rather than the propositional language, $\mathcal{P} \mathcal{L}(S)$, used earlier in this paper.

Thus, let $\mathcal{L}(S)$ be the local language for a system $S$. As explained in paper I, this is a typed language whose minimal set of ground-type symbols is $\Sigma$ and $\mathcal{R}$. There is a non-empty set, $F_{\mathcal{L}(S)}(\Sigma, \mathcal{R})$, of function symbols $A: \Sigma \rightarrow \mathcal{R}$ that correspond to the physical quantities of $S$.

Now consider a representation, $\phi$, of $\mathcal{L}(S)$ in a topos $\tau_{\phi}$. As discussed in paper I, the propositional aspects of the language $\mathcal{L}(S)$ are captured in the term ' $A(\tilde{s}) \in \tilde{\Delta}$ ' of type $\Omega$, where $\tilde{s}$ and $\tilde{\Delta}$ are variables of type $\Sigma$ and $P \mathcal{R}$ respectively [1. In a topos representation, $\phi$, the representation, $\llbracket A(\tilde{s}) \in \tilde{\Delta} \rrbracket_{\phi}$, of the term ' $A(\tilde{s}) \in \tilde{\Delta}$ ' is given by 
the chain of arrow 21$] 5$

$$
\Sigma_{\phi} \times P \mathcal{R}_{\phi} \stackrel{A_{\phi} \times \mathrm{id}}{\longrightarrow} \mathcal{R}_{\phi} \times P \mathcal{R}_{\phi} \stackrel{{ }^{e_{\mathcal{R}_{\phi}}}}{\longrightarrow} \Omega_{\tau_{\phi}}
$$

in the topos $\tau_{\phi}$. Then, if $\ulcorner\Xi\urcorner: 1_{\tau_{\phi}} \rightarrow P \mathcal{R}_{\phi}$ is a sub-object of the quantity-value object $\mathcal{R}_{\phi}$, we get the chain

$$
\Sigma_{\phi} \simeq \Sigma_{\phi} \times 1_{\tau_{\phi}} \stackrel{\mathrm{id} \times\ulcorner\Xi\urcorner}{\longrightarrow} \Sigma_{\phi} \times P \mathcal{R}_{\phi} \stackrel{A_{\phi} \times \mathrm{id}}{\longrightarrow} \mathcal{R}_{\phi} \times P \mathcal{R}_{\phi} \stackrel{e_{\mathcal{R}_{\phi}}}{\longrightarrow} \Omega_{\tau_{\phi}}
$$

which is interpreted as the characteristic arrow associated with the proposition " $A \varepsilon \Xi$ ".

Equivalently, we can use the term, $\{\tilde{s} \mid A(\tilde{s}) \in \tilde{\Delta}\}$, which has a free variable $\tilde{\Delta}$ of type $P \mathcal{R}$ and is of type $P \Sigma$ (see paper I, [1]). This term is represented by the arrow $\llbracket\{\tilde{s} \mid A(\tilde{s}) \in \tilde{\Delta}\} \rrbracket_{\phi}: P \mathcal{R}_{\phi} \rightarrow P \Sigma_{\phi}$, which is the power transpose of $\llbracket A(\tilde{s}) \in \tilde{\Delta} \rrbracket_{\phi}$ :

$$
\llbracket\{\tilde{s} \mid A(\tilde{s}) \in \tilde{\Delta}\} \rrbracket_{\phi}=\left\ulcorner\llbracket A(\tilde{s}) \in \tilde{\Delta} \rrbracket_{\phi}\right\urcorner
$$

The proposition " $A \varepsilon \Xi$ " is then represented by the arrow $\llbracket\{\tilde{s} \mid A(\tilde{s}) \in \tilde{\Delta}\} \rrbracket_{\phi} \circ\ulcorner\Xi\urcorner$ : $1_{\tau_{\phi}} \rightarrow P \mathcal{R}_{\phi}$.

We note an important difference with the analogous situation for the language $\mathcal{P} \mathcal{L}(S)$. In propositions of the type " $A \varepsilon \Delta$ ", the symbol ' $\Delta$ ' is external to the language (it is a specific subset of $\mathbb{R}$ ), and it is independent of the representation of $\mathcal{P} \mathcal{L}(S)$. However, in the case of $\mathcal{L}(S)$, the variable $\tilde{\Delta}$ is internal to the language, and the quantity $\Xi$ in the proposition " $A \varepsilon \Xi$ " is a sub-object of $\mathcal{R}_{\phi}$ in a specific topos representation, $\phi$, of $\mathcal{L}(S)$.

This is how physical propositions are represented mathematically. But how are truth values to be assigned to these propositions? In the topos $\tau_{\phi}$ a truth value is an element of the Heyting algebra $\Gamma \Omega_{\tau_{\phi}}$. Thus the challenge is to assign a global element of $\Omega_{\tau_{\phi}}$ to each proposition associated with the representation of the term $\{\tilde{s} \mid A(\tilde{s}) \in \tilde{\Delta}\}$ of type $P \Sigma$; (or, equivalently, the representation of the term ' $A(\tilde{s}) \in \tilde{\Delta}^{\prime}$ ).

Let us first pose this question at a linguistic level. In a representation $\phi$, an element of $\Gamma \Omega_{\tau_{\phi}}$ is associated with a representation of a term of type $\Omega$ with no free variables. Hence the question can be rephrased as asking how a term, $t$, in $\mathcal{L}(S)$ of type $P \Sigma$ can be 'converted' into a term of type $\Omega$ ? At this stage, we are happy to have free variables in this term, in which case it will be represented by an arrow in $\tau_{\phi}$ with co-domain $\Omega_{\tau_{\phi}}$, but whose domain is other than $1_{\tau_{\phi}}$. This is an intermediate stage to obtaining a global element of $\Omega_{\tau_{\phi}}$.

In the context of the language $\mathcal{L}(S)$ there are two obvious ways of 'converting' the term $t$ of type $P \Sigma$ to a term of type $\Omega$ :

1. Choose a term, $s$, of type $\Sigma$; then the term ' $s \in t$ ' is of type $\Omega$.

2. Choose a term, $\mathbb{T}$, of type $P P \Sigma$; then the term ' $t \in \mathbb{T}$ ' is of type $\Omega$.

\footnotetext{
${ }^{21} \operatorname{In}$ (4.7), $e_{\mathcal{R}_{\phi}}: \mathcal{R}_{\phi} \times P \mathcal{R}_{\phi} \rightarrow \Omega_{\tau_{\phi}}$ is the evaluation arrow associated with the power object $P \mathcal{R}_{\phi}$.
} 
In regard to the first option, the simplest term of type $\Sigma$ is a variable $\tilde{s_{1}}$ of type $\Sigma$. Then, the term ' $\tilde{s_{1}} \in\{\tilde{s} \mid A(\tilde{s}) \in \tilde{\Delta}\}$ ' is of type $\Omega$ with the free variables $\tilde{s_{1}}$ of type $\Sigma$ and $\tilde{\Delta}$ of type $P \mathcal{R}$. However, the axiom of comprehension in $\mathcal{L}(S)$ says that

$$
\tilde{s_{1}} \in\{\tilde{s} \mid A(\tilde{s}) \in \tilde{\Delta}\} \Leftrightarrow A\left(\tilde{s_{1}}\right) \in \tilde{\Delta}
$$

and so we are back with the term ' $A(\tilde{s}) \in \tilde{\Delta}$ ', which is of type $\Omega$ and with the free variable $\tilde{s}$ of type $\Sigma$.

As stated above, the $\phi$-representation, $\llbracket A(\tilde{s}) \in \tilde{\Delta} \rrbracket_{\phi}$, of ' $A(\tilde{s}) \in \tilde{\Delta}$ ' is the chain of arrows in (4.7). Now, if the representation, $\phi$, is such that there exist global elements, $\ulcorner s\urcorner: 1_{\tau_{\phi}} \rightarrow \Sigma_{\phi}$, of $\Sigma_{\phi}$, then each such element can be regarded as a '(micro)-state' of the system. This can be combined with the arrow $\llbracket A(\tilde{s}) \in \tilde{\Delta} \rrbracket_{\phi}: \Sigma_{\phi} \times P \mathcal{R}_{\phi} \rightarrow \Omega_{\tau_{\phi}}$ to give the arrow

$$
\llbracket A(\tilde{s}) \in \tilde{\Delta} \rrbracket_{\phi} \circ(\ulcorner s\urcorner \times \mathrm{id}): 1_{\tau_{\phi}} \times P \mathcal{R}_{\phi} \rightarrow \Sigma_{\phi} \times P \mathcal{R}_{\phi} \rightarrow \Omega_{\tau_{\phi}}
$$

Finally, if $\ulcorner\Xi\urcorner: 1_{\tau_{\phi}} \rightarrow P \mathcal{R}_{\phi}$ is a sub-object of the quantity-value object $\mathcal{R}_{\phi}$, then

$$
\llbracket A(\tilde{s}) \in \tilde{\Delta} \rrbracket_{\phi} \circ(\ulcorner s\urcorner \times \mathrm{id}) \circ\ulcorner\Xi\urcorner: 1_{\tau_{\phi}} \rightarrow \Omega_{\tau_{\phi}}
$$

is the desired global element of $\Omega_{\tau_{\phi}}$. To simplify the notation somewhat, we will rewrite (4.12) as

$$
\llbracket A(\tilde{s}) \in \tilde{\Delta} \rrbracket_{\phi}\langle\ulcorner s\urcorner,\ulcorner\Xi\urcorner\rangle: 1_{\tau_{\phi}} \rightarrow \Omega_{\tau_{\phi}}
$$

Thus, $\langle\ulcorner s\urcorner,\ulcorner\Xi\urcorner\rangle: 1_{\tau_{\phi}} \rightarrow \Sigma_{\phi} \times P \mathcal{R}_{\phi}$.

In other words, when the 'state of the system' is $s \in \Gamma \Sigma_{\phi}$, the 'truth value' of the proposition " $A \varepsilon \Xi$ " - as represented by the sub-object of $\Sigma_{\phi}$ with characteristic arrow $\llbracket A(\tilde{s}) \in \tilde{\Delta} \rrbracket_{\phi} \circ($ id $\circ\ulcorner\Xi\urcorner): \Sigma_{\phi} \times 1 \rightarrow \Omega_{\tau_{\phi}}$-is the global element of $\Omega_{\tau_{\phi}}$ defined by $\llbracket A(\tilde{s}) \in \tilde{\Delta} \rrbracket_{\phi}\langle\ulcorner s\urcorner,\ulcorner\Xi\urcorner\rangle: 1_{\tau_{\phi}} \rightarrow \Omega_{\tau_{\phi}}$. All this may seem rather complicated but, in fact, it is quite straightforward once one gets used to the notation.

This is the procedure that is adopted in classical physics, where a truth value is assigned to propositions by specifying a micro-state, $s \in \Sigma_{\sigma}$, where $\Sigma_{\sigma}$ is the classical state space in the representation $\sigma$ of $\mathcal{L}(S)$. Specifically, for all $s \in \Sigma_{\sigma}$, the truth value of the proposition " $A \varepsilon \Delta$ " as given by (4.13), is (c.f. (4.1))

$$
\nu(A \varepsilon \Delta ; s)=\llbracket A(\tilde{s}) \in \tilde{\Delta} \rrbracket_{\sigma}(s, \Delta)= \begin{cases}1 & \text { if } A_{\sigma}(s) \in \Delta ; \\ 0 & \text { otherwise. }\end{cases}
$$

where $\llbracket A(\tilde{s}) \in \tilde{\Delta} \rrbracket_{\sigma}: \Sigma_{\sigma} \times P \mathbb{R} \rightarrow \Omega_{\tau_{\sigma}} \simeq\{0,1\}$. Thus we recover the earlier result (4.3).

\subsubsection{Truth Objects in a General Topos.}

By hindsight, we know that the option to use global elements of $\Sigma_{\phi}$ is not available in the quantum case. There, the state object, $\underline{\Sigma}$, is the spectral presheaf, and this 
has no global elements by virtue of the Kochen-Specker theorem. The absence of global elements of the state object $\Sigma_{\phi}$ could well be true in many other topos models of physics, and therefore an alternative general strategy is needed to that employing micro-states $\ulcorner s\urcorner: 1_{\tau_{\phi}} \rightarrow \Sigma_{\phi}$.

This takes us to the second possibility: namely, to introduce a term, $\mathbb{T}$, of type $P P \Sigma$, and then work with the term ' $\{\tilde{s} \mid A(\tilde{s}) \in \tilde{\Delta}\} \in \mathbb{T}$ ', which is of type $\Omega$, and has whatever free variables are contained in $\mathbb{T}$, plus the variable $\tilde{\Delta}$ of type $P \mathcal{R}$.

The simplest choice is to let the term of type $P P \Sigma$ be a variable, $\tilde{\mathbb{T}}$, of type $P P \Sigma$, in which case the term ' $\{\tilde{s} \mid A(\tilde{s}) \in \tilde{\Delta}\} \in \tilde{\mathbb{T}}$ ' has variables $\tilde{\Delta}$ of type $P \mathcal{R}$ and $\tilde{\mathbb{T}}$ of type $P P \Sigma$. Therefore, in a topos representation it is represented by an arrow $\llbracket\{\tilde{s} \mid A(\tilde{s}) \in \tilde{\Delta}\} \in \tilde{\mathbb{T}} \rrbracket_{\phi}: P \mathcal{R}_{\phi} \times P\left(P \Sigma_{\phi}\right) \rightarrow \Omega_{\tau_{\phi}}$. In detail (see [5]) we have that

$$
\llbracket\{\tilde{s} \mid A(\tilde{s}) \in \tilde{\Delta}\} \in \tilde{\mathbb{T}} \rrbracket_{\phi}=e_{P \Sigma_{\phi}} \circ\left\langle\llbracket\{\tilde{s} \mid A(\tilde{s}) \in \tilde{\Delta}\} \rrbracket_{\phi}, \llbracket \tilde{\mathbb{T}} \rrbracket_{\phi}\right\rangle
$$

where $e_{P \Sigma_{\phi}}: P \Sigma_{\phi} \times P\left(P \Sigma_{\phi}\right) \rightarrow \Omega_{\tau_{\phi}}$ is the usual evaluation arrow. In using this expression we need the $\phi$-representatives:

$$
\begin{aligned}
& \llbracket\{\tilde{s} \mid A(\tilde{s}) \in \tilde{\Delta}\} \rrbracket_{\phi}: P \mathcal{R}_{\phi} \quad \rightarrow \quad P \Sigma_{\phi} \\
& \llbracket \tilde{\mathbb{T}} \rrbracket_{\phi}: P\left(P \Sigma_{\phi}\right) \quad \stackrel{\mathrm{id}}{\longrightarrow} P\left(P \Sigma_{\phi}\right)
\end{aligned}
$$

Finally, let $\langle\ulcorner\Xi\urcorner,\ulcorner\mathbb{T}\urcorner\rangle$ be a pair of global elements in $P \mathcal{R}_{\phi}$ and $P\left(P \Sigma_{\phi}\right)$ respectively, so that $\ulcorner\Xi\urcorner: 1_{\tau_{\phi}} \rightarrow P \mathcal{R}_{\phi}$ and $\ulcorner\mathbb{T}\urcorner: 1_{\tau_{\phi}} \rightarrow P\left(P \Sigma_{\phi}\right)$. Thus, $\ulcorner\mathbb{T}\urcorner$ is a concrete truth object in $\tau_{\phi}$. Then, for the physical proposition " $A \varepsilon \Xi$ ", we have the truth value

$$
\nu(A \varepsilon \Xi ; \mathbb{T})=\llbracket\{\tilde{s} \mid A(\tilde{s}) \in \tilde{\Delta}\} \in \tilde{\mathbb{T}} \rrbracket_{\phi}\langle\ulcorner\Xi\urcorner,\ulcorner\mathbb{T}\urcorner\rangle: 1_{\tau_{\phi}} \rightarrow \Omega_{\tau_{\phi}}
$$

A small generalisation. More generally, if $\tilde{K}$ and $\tilde{\mathbb{T}}$ are variables of type $P \Sigma$ and $P(P \Sigma)$ respectively, the term of interest is ' $\tilde{K} \in \tilde{\mathbb{T}}$ '. In the representation, $\phi$, of $\mathcal{L}(S)$, this term maps to an arrow $\llbracket \tilde{K} \in \tilde{\mathbb{T}} \rrbracket_{\phi}: P \Sigma_{\phi} \times P\left(P \Sigma_{\phi}\right) \rightarrow \Omega_{\tau_{\phi}}$. Here,

$$
\llbracket \tilde{K} \in \tilde{\mathbb{T}} \rrbracket_{\phi}=e_{P \Sigma_{\phi}} \circ\left\langle\llbracket \tilde{K} \rrbracket_{\phi}, \llbracket \tilde{\mathbb{T}} \rrbracket_{\phi}\right\rangle
$$

where

$$
\begin{aligned}
\llbracket \tilde{K} \rrbracket_{\phi}: P \Sigma_{\phi} & \stackrel{\mathrm{id}}{\longrightarrow} P \Sigma_{\phi} \\
\llbracket \tilde{\mathbb{T}} \rrbracket_{\phi}: P\left(P \Sigma_{\phi}\right) & \stackrel{\mathrm{id}}{\longrightarrow} P\left(P \Sigma_{\phi}\right)
\end{aligned}
$$

Let $\ulcorner K\urcorner,\ulcorner\mathbb{T}\urcorner$ be global elements of $P \Sigma_{\phi}$ and $P\left(P \Sigma_{\phi}\right)$ respectively, so that $\ulcorner K\urcorner$ : $1_{\tau_{\phi}} \rightarrow P \Sigma_{\phi}$ and $\ulcorner\mathbb{T}\urcorner: 1_{\tau_{\phi}} \rightarrow P\left(P \Sigma_{\phi}\right)$. We adopt the notation $\langle\ulcorner K\urcorner,\ulcorner\mathbb{T}\urcorner\rangle: 1_{\tau_{\phi}} \rightarrow$ $P \Sigma_{\phi} \times P\left(P \Sigma_{\phi}\right)$. Then the truth of the (mathematical) proposition " $\ulcorner K\urcorner \in \mathbb{T}$ " is

$$
\begin{aligned}
\nu(\ulcorner K\urcorner \in \mathbb{T}) & =\llbracket \tilde{K} \in \tilde{\mathbb{T}} \rrbracket_{\phi}\langle\ulcorner K\urcorner,\ulcorner\mathbb{T}\urcorner\rangle \\
& =e_{P \Sigma_{\phi}} \circ\langle\ulcorner K\urcorner,\ulcorner\mathbb{T}\urcorner\rangle: 1_{\tau_{\phi}} \rightarrow \Omega_{\tau_{\phi}}
\end{aligned}
$$




\subsubsection{The Example of Classical Physics.}

If classical physics is studied this way, the general formalism simplifies, and the term ' $\{\tilde{s} \mid A(\tilde{s}) \in \tilde{\Delta}\} \in \tilde{\mathbb{T}}$ ' is represented by the function $\llbracket\{\tilde{s} \mid A(\tilde{s}) \in \tilde{\Delta}\} \in \tilde{\mathbb{T}} \rrbracket_{\sigma}$ : $P \mathbb{R} \times P\left(P \Sigma_{\sigma}\right) \rightarrow \Omega_{\text {Sets }} \simeq\{0,1\}$ defined by

$$
\begin{aligned}
\nu(A \varepsilon \Delta ; \mathbb{T})=\llbracket\{\tilde{s} \mid A(\tilde{s}) \in \tilde{\Delta}\} \in \tilde{\mathbb{T}} \rrbracket_{\sigma}(\Delta, \mathbb{T}) & = \begin{cases}1 & \text { if }\left\{s \in \Sigma_{\sigma} \mid A_{\sigma}(s) \in \Delta\right\} \in \mathbb{T} \\
0 & \text { otherwise }\end{cases} \\
& = \begin{cases}1 & \text { if } A_{\sigma}^{-1}(\Delta) \in \mathbb{T} \\
0 & \text { otherwise }\end{cases}
\end{aligned}
$$

for all $\mathbb{T} \in P\left(P \Sigma_{\sigma}\right)$. We can clearly see the sense in which the truth object $\mathbb{T}$ is playing the role of a state. Note that the result (4.23) of classical physics is a special case of (4.18).

To recover the usual truth values given in (4.14), a truth object, $\mathbb{T}^{s}$, must be associated with each micro-state $s \in \Sigma_{\sigma}$. The correct choice is

$$
\mathbb{T}^{s}:=\left\{K \subseteq \Sigma_{\sigma} \mid s \in K\right\}
$$

for each $s \in \Sigma_{\sigma}$. It is clear that $s \in A_{\sigma}^{-1}(\Delta)$ (or, equivalently, $A_{\sigma}(s) \in \Delta$ ) if, and only if, $A_{\sigma}^{-1}(\Delta) \in \mathbb{T}^{s}$. Hence (4.23) can be rewritten as

$$
\nu\left(A \varepsilon \Delta ; \mathbb{T}^{s}\right):= \begin{cases}1 & \text { if } s \in A_{\sigma}^{-1}(\Delta) ; \\ 0 & \text { otherwise. }\end{cases}
$$

which reproduces (4.14) once $\nu(A \varepsilon \Delta ; s)$ is identified with $\nu\left(A \varepsilon \Delta ; \mathbb{T}^{s}\right)$.

\subsubsection{The Truth Object $\underline{\mathbb{T}}^{|\psi\rangle}$ in Quantum Theory}

We can now start to discuss the application of these ideas to quantum theory. In order to use (4.22) or (4.18) we need to construct a concrete truth object, $\ulcorner\mathbb{T}\urcorner$, in the topos $\tau_{\phi}:=\operatorname{Sets}^{\mathcal{V}(\mathcal{H})^{\mathrm{op}}}$. Thus $\ulcorner\underline{\mathbb{T}}\urcorner: 1_{\tau_{\phi}} \rightarrow P(P \underline{\Sigma})$; equivalently, $\underline{\mathbb{T}}$ is a sub-object of $P \underline{\Sigma}$.

However, we have to keep in mind the need to restrict to clopen sub-objects of $\underline{\Sigma}$. In particular, we have to show that there is a well-defined presheaf $P_{\mathrm{cl}} \underline{\Sigma}$ such that

$$
\operatorname{Sub}_{\mathrm{cl}}(\underline{\Sigma}) \simeq \Gamma\left(P_{\mathrm{cl}} \underline{\Sigma}\right)
$$

We will prove this shortly in Section 4.3 ,

Given (4.26), $\underline{K} \in \operatorname{Sub}_{\mathrm{cl}}(\underline{\Sigma})$ is equivalent to an arrow $\ulcorner\underline{K}\urcorner: 1_{\tau_{\phi}} \rightarrow P_{\mathrm{cl}} \underline{\Sigma}$; and hence a truth object, $\underline{\mathbb{T}}$, has to be a sub-object of $P_{\mathrm{cl}} \underline{\underline{ }}$ in order that the valuation $\nu(\ulcorner K\urcorner \in \mathbb{T})$ in (4.22) is meaningful.

This truth value, $\nu(\ulcorner K\urcorner \in \mathbb{T})$, is a global element of $\underline{\Omega}$, and in the topos of

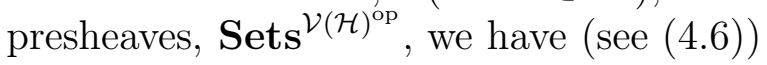

$$
\nu(\ulcorner\underline{K}\urcorner \in \underline{\mathbb{T}})_{V}:=\left\{V^{\prime} \subseteq V \mid \underline{K}_{V^{\prime}} \in \underline{\mathbb{T}}_{V^{\prime}}\right\}
$$


for each context $V$.

There are various examples of $\underline{K}$ that are of interest to us. In particular, let $\underline{K}=$ $\delta(\hat{P})$ for some projector $\hat{P}$. Then, using the propositional language $\mathcal{P} \mathcal{L}(S)$ discussed earlier in this paper, the 'truth' of the proposition represented by $\hat{P}$ (for example, "A $\varepsilon \Delta ")$ is

$$
\left.\nu(\ulcorner\delta(\hat{P})\urcorner \in \underline{\mathbb{T}})_{V}=\left\{V^{\prime} \subseteq V \mid \delta(\hat{P})_{V^{\prime}}\right) \in \underline{\mathbb{T}}_{V^{\prime}}\right\}
$$

for all stages $V$.

In the case of the local language $\mathcal{L}(S)$, the important example is when the subobject $\underline{K}$ of $\underline{\Sigma}$ is of the form $A_{\phi}^{-1}(\underline{\Xi})$, for some sub-object $\Xi$ of $\underline{R}$. This will yield the truth value, $\nu(A \varepsilon \Xi ; \underline{\mathbb{T}})$, in (4.18). However, to discuss this further requires the representation of function symbols $A: \Sigma \rightarrow \mathcal{R}$ in the topos $\operatorname{Sets}^{\mathcal{V}(\mathcal{H})^{\mathrm{op}}}$, and this is deferred until paper III [2].

The definition of the truth objects $\underline{\mathbb{T}}^{|\psi\rangle}$. The definition of truth objects in quantum theory was studied in the original papers [13, 14, 15, 16]. It was shown there that to each quantum state $|\psi\rangle \in \mathcal{H}$, there corresponds a truth object, $\underline{\mathbb{T}}^{|\psi\rangle}$, which is defined as the following sub-object of the outer presheaf, $\underline{O}$ :

$$
\begin{aligned}
\underline{\mathbb{T}}_{V}^{|\psi\rangle} & :=\left\{\hat{\alpha} \in \underline{O}_{V} \mid \operatorname{Prob}(\hat{\alpha} ;|\psi\rangle)=1\right\} \\
& =\left\{\hat{\alpha} \in \underline{O}_{V} \mid\langle\psi|\hat{\alpha}| \psi\rangle=1\right\}
\end{aligned}
$$

for all stages $V \in \operatorname{Ob}(\mathcal{V}(\mathcal{H}))$. Here, $\operatorname{Prob}(\hat{\alpha} ;|\psi\rangle)$ is the usual expression for the probability that the proposition represented by the projector $\hat{\alpha}$ is true, given that the quantum state is the (normalised) vector $|\psi\rangle$.

It is easy to see that (4.29) defines a genuine sub-object $\underline{\mathbb{T}}^{|\psi\rangle}=\left\{\underline{\mathbb{T}}_{V}^{|\psi\rangle} \mid V \in\right.$ $\operatorname{Ob}(\mathcal{V}(\mathcal{H}))\}$ of $\underline{O}$, since if $\hat{\beta} \succeq \hat{\alpha}$, then $\langle\psi|\hat{\beta}| \psi\rangle \geq\langle\psi|\hat{\alpha}| \psi\rangle$. Therefore, if $V^{\prime} \subseteq V$ and $\hat{\alpha} \in \underline{O}_{V}$, then $\left\langle\psi\left|\underline{O}\left(i_{V^{\prime} V}\right)(\hat{\alpha})\right| \psi\right\rangle \geq\langle\psi|\hat{\alpha}| \psi\rangle$. In particular, if $\langle\psi|\hat{\alpha}| \psi\rangle=1$ then $\left\langle\psi\left|\underline{O}\left(i_{V^{\prime} V}\right)(\hat{\alpha})\right| \psi\right\rangle=1$.

We note that there is an interesting connection between $\mathbb{T}^{|\psi\rangle}$ and (outer) daseinisation. Specifically, at each stage $V$, take the smallest projection $\hat{\alpha}_{0}(V)$ in $\mathbb{T}_{V}^{|\psi\rangle}$. Then, clearly, since $\langle\psi|\hat{\alpha}| \psi\rangle=1$ just means $\hat{\alpha} \succeq \hat{P_{|\psi\rangle}}$, we have

$$
\hat{\alpha}_{0}(V)=\delta\left(\hat{P}_{|\psi\rangle}\right)_{V}
$$

where $\hat{P}_{|\psi\rangle}$ is the projection onto the one-dimensional subspace generated by $|\psi\rangle$.

The next step is to define the presheaf $P_{\mathrm{cl}} \underline{\Sigma}$, and then show that there is a monic arrow $\underline{O} \rightarrow P_{\mathrm{cl}} \underline{\Sigma}$, so that $\underline{O}$ is a sub-object of $P_{\mathrm{cl}} \underline{\Sigma}$. Then, since $\underline{\mathbb{T}}^{|\psi\rangle}$ is a sub-object of $\underline{O}$, and $\underline{O}$ is a sub-object of $P_{\mathrm{cl}} \underline{\Sigma}$, it follows that $\underline{\mathbb{T}}^{|\psi\rangle}$ is a sub-object of $P_{\mathrm{cl}} \underline{\Sigma}$, as required.

With this definition of $\underline{\mathbb{T}}^{|\psi\rangle}$, the truth value, (4.28), for the propositional language $\mathcal{P} \mathcal{L}(S)$ becomes

$$
\nu\left(\ulcorner\delta(\hat{P})\urcorner \in \underline{\mathbb{T}}^{|\psi\rangle}\right)_{V}=\left\{V^{\prime} \subseteq V \mid\left\langle\psi\left|\delta(\hat{P})_{V^{\prime}}\right| \psi\right\rangle=1\right\}
$$


It is easy to see that the definition of a truth object in (4.29) can be extended to a mixed state with a density-matrix operator $\hat{\rho}$ : simply replace the definition in (4.29) with

$$
\begin{aligned}
\underline{\mathbb{T}}_{V}^{\hat{\rho}} & :=\left\{\hat{\alpha} \in \underline{O}_{V} \mid \operatorname{Prob}(\hat{\alpha} ; \rho)=1\right\} \\
& =\left\{\hat{\alpha} \in \underline{O}_{V} \mid \operatorname{tr}(\hat{\rho} \hat{\alpha})=1\right\}
\end{aligned}
$$

However there is an important difference between the truth object associated with a vector state, $|\psi\rangle$, and the one associated with a density matrix, $\rho$. In the vector case, it is easy to see that the mapping $|\psi\rangle \rightarrow \underline{\mathbb{T}}^{|\psi\rangle}$ is one-to-one (up to a phase factor on $|\psi\rangle$ ) so that, in principle, the state $|\psi\rangle$ can be recovered from $\underline{\mathbb{T}}^{|\psi\rangle}$ (up to a phase-factor). On the other hand, there are simple counterexamples which show that, in general, the density matrix, $\rho$ cannot be recovered from $\underline{\mathbb{T}}^{\hat{\rho}}$.

In a sense, this should not surprise us. The analogue of a density matrix in classical physics is a probability measure $\mu$ defined on the classical state space $\mathcal{S}$. Individual microstates $s \in \mathcal{S}$ are in one-to-one correspondence with probability measures of the form $\mu_{s}$ defined by $\mu_{s}(K)=1$ if $s \in K, \mu_{s}(K)=0$ if $s \notin K$.

However, one of the main claims of our programme is that any theory 'looks like' classical physics in the appropriate topos. This suggests that, in the topos version of quantum theory, a density matrix should be represented by some sort of measure on the state object $\underline{\Sigma}$ in the topos $\tau_{\phi}$; and this should relate in some way to an 'integral' of 'vector truth objects'. The development of this idea is one of the many interesting tasks for the future.

\subsubsection{Time-dependence and the Truth Object.}

As emphasised at the end of the first paper in this series [1, the question of time dependence depends on the theory-type being considered. The structure of the language $\mathcal{L}(S)$ that has been used so far is such that the time variable lies outside the language. In this situation, the time dependence of the system can be implemented in several ways.

For example, we can make the truth object time dependent, giving a family of truth objects, $t \mapsto \underline{\mathbb{T}}^{t}, t \in \mathbb{R}$. In the case of classical physics, with the truth objects $\underline{\mathbb{T}}^{s}$, $s \in \Sigma_{\sigma}$, the time evolution comes from the time dependence, $t \mapsto s_{t}$, of the microstate in accordance with the classical equations of motion. This gives the family $t \mapsto \underline{\mathbb{T}}^{s t}$ of truth objects.

Something similar happens in quantum theory, with a family $t \mapsto \underline{\mathbb{T}}^{|\psi\rangle_{t}}$ of truth objects, where the states $|\psi\rangle_{t}$ satisfy the time-dependent Schrödinger equation. Thus both classical and quantum truth objects belong to a 'Schödinger picture' of time evolution.

It is also possible to construct a 'Heisenberg picture' where the truth object is constant but the physical quantities and associated propositions are time dependent. We will return to this in the next paper when we discuss the use of unitary operators. 


\subsection{The Presheaf $P_{\mathrm{cl}} \underline{\Sigma}$}

\subsubsection{The Definition of $P_{\mathrm{cl}} \underline{\Sigma}$.}

We must now show that there really is a presheaf $P_{\mathrm{cl}} \underline{\Sigma}$.

The easiest way of defining $P_{\mathrm{cl}} \underline{\Sigma}$ is to start with the concrete expression for the normal power object $P \underline{\Sigma}[\underline{6}$. First, if $\underline{F}$ is any presheaf over $\mathcal{V}(\mathcal{H})$, define the restriction of $\underline{F}$ to $V$ to be the functor $\underline{F} \downarrow V$ from the category $\iota^{22} \downarrow V$ to Sets that assigns to each $V_{1} \subseteq V$, the set $\underline{F}_{V_{1}}$, and with the obvious induced presheaf maps.

Then, at each stage $V, P \underline{\Sigma}_{V}$ is the set of natural transformations from $\underline{\Sigma} \downarrow V$ to $\underline{\Omega} \downarrow V$. These are in one-to-one correspondence with families of maps $\sigma:=\left\{\sigma_{V_{1}}: \underline{\Sigma}_{V_{1}} \rightarrow\right.$ $\left.\underline{\Omega}_{V_{1}} \mid V_{1} \subseteq V\right\}$, with the following commutative diagram for all $V_{2} \subseteq V_{1} \subseteq V$ :

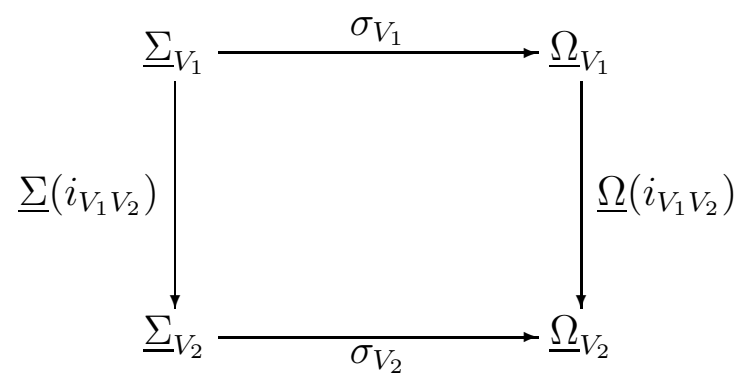

The presheaf maps are defined by

$$
\begin{aligned}
P \underline{\Sigma}\left(i_{V_{1} V}\right): P \underline{\Sigma}_{V} & \rightarrow P \underline{\Sigma}_{V_{1}} \\
\sigma & \mapsto\left\{\sigma_{V_{2}} \mid V_{2} \subseteq V_{1}\right\}
\end{aligned}
$$

and the evaluation arrow ev $: P \underline{\Sigma} \times \underline{\Sigma} \rightarrow \underline{\Omega}$, has the form, at each stage $V$ :

$$
\begin{aligned}
\mathrm{ev}_{V}: P \underline{\Sigma}_{V} \times \underline{\Sigma}_{V} & \rightarrow \underline{\Omega}_{V} \\
(\sigma, \lambda) & \mapsto \sigma_{V}(\lambda)
\end{aligned}
$$

Moreover, in general, given a map $\chi: \underline{\Sigma}_{V} \rightarrow \underline{\Omega}_{V}$, the subset of $\underline{\Sigma}_{V}$ associated with the corresponding sub-object is $\chi^{-1}(1)$, where 1 is the unit ('truth') in the Heyting algebra $\underline{\Omega}_{V}$.

This suggests strongly that an object, $P_{\mathrm{cl}} \underline{\Sigma}$, in $\operatorname{Sets}^{\mathcal{V}(\mathcal{H})^{\mathrm{op}}}$ can be defined using the same definition of $P \underline{\Sigma}$ as above, except that the family of maps $\sigma:=\left\{\sigma_{V_{1}}: \underline{\Sigma}_{V_{1}} \rightarrow\right.$ $\left.\underline{\Omega}_{V_{1}} \mid V_{1} \subseteq V\right\}$ must be such that, for all $V_{1} \subseteq V, \sigma_{V_{1}}^{-1}(1)$ is a clopen subset of the (extremely disconnected) Hausdorff space $\underline{\Sigma}_{V_{1}}$. It is straightforward to check that such a restriction is consistent, and that $\operatorname{Sub}_{\mathrm{cl}}(\underline{\Sigma}) \simeq \Gamma\left(P_{\mathrm{cl}} \underline{\Sigma}\right)$ as required.

\subsubsection{The Monic Arrow From $\underline{O}$ to $P_{\mathrm{cl}} \underline{\Sigma}$.}

We define $\iota: \underline{O} \times \underline{\Sigma} \rightarrow \underline{\Omega}$, with the power transpose $\ulcorner\iota\urcorner: \underline{O} \rightarrow P_{\mathrm{cl}} \underline{\Sigma}$, as follows. First recall that in any topos, $\tau$ there is a bijection $\operatorname{Hom}_{\tau}\left(A, C^{B}\right) \simeq \operatorname{Hom}_{\tau}(A \times B, C)$, and

\footnotetext{
${ }^{22}$ The notation $\downarrow V$ means the partially-ordered set of all subalgebras $V^{\prime} \subseteq V$.
} 
hence, in particular, (using $\left.P \underline{\Sigma}=\underline{\Omega^{\underline{\Sigma}}}\right)$

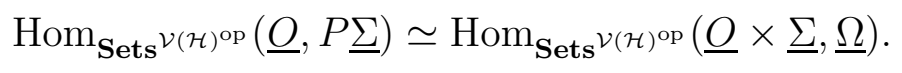

Now let $\hat{\alpha} \in \mathcal{P}(V)$, and let $S_{\hat{\alpha}}:=\left\{\lambda \in \underline{\Sigma}_{V} \mid \lambda(\hat{\alpha})=1\right\}$ be the clopen subset of $\underline{\Sigma}_{V}$ that corresponds to the projector $\hat{\alpha}$ via the spectral theorem; see (2.23). Then we define $\iota: \underline{O} \times \underline{\Sigma} \rightarrow \underline{\Omega}$ at stage $V$ by

$$
\iota_{V}(\hat{\alpha}, \lambda):=\left\{V^{\prime} \subseteq V \mid \underline{\Sigma}\left(i_{V^{\prime} V}\right)(\lambda) \in S_{\underline{Q}\left(i_{V^{\prime} V}\right)(\hat{\alpha})}\right\}
$$

for all $(\hat{\alpha}, \lambda) \in \underline{O}_{V} \times \underline{\Sigma}_{V}$.

On the other hand, the basic result relating coarse-graining to subsets of $\underline{\Sigma}$ is

$$
S_{\underline{Q}\left(i_{\left.V^{\prime} V\right)}\left(\delta(\hat{\alpha})_{V}\right)\right.}=\underline{\Sigma}\left(i_{V^{\prime} V}\right)\left(S_{\delta(\hat{\alpha})_{V}}\right)
$$

for all $V^{\prime} \subseteq V$ and for all $\hat{\alpha} \in \underline{O}_{V}$. It follows that

$$
\iota_{V}(\hat{\alpha}, \lambda):=\left\{V^{\prime} \subseteq V \mid \underline{\Sigma}\left(i_{V^{\prime} V}\right)(\lambda) \in \underline{\Sigma}\left(i_{V^{\prime} V}\right)\left(S_{\hat{\alpha}}\right)\right\}
$$

for all $(\hat{\alpha}, \lambda) \in \underline{O}_{V} \times \underline{\Sigma}_{V}$. In this form is is clear that $\iota_{V}(\hat{\alpha}, \lambda)$ is indeed a sieve on $V$; i.e., an element of $\underline{\Omega}_{V}$.

The next step is to show that the collection of maps $\iota_{V}: \underline{O}_{V} \times \underline{\Sigma}_{V} \rightarrow \underline{\Omega}_{V}$ defined in (4.38) constitutes a natural transformation from the object $\underline{O} \times \underline{\underline{\Sigma}}$ to the object $\underline{\Omega}$ in the topos $\operatorname{Sets}^{\mathcal{V}(\mathcal{H})^{\mathrm{op}}}$. This involves chasing around a few commutative squares, and we will spare the reader the ordeal. There is some subtlety, since we really want to

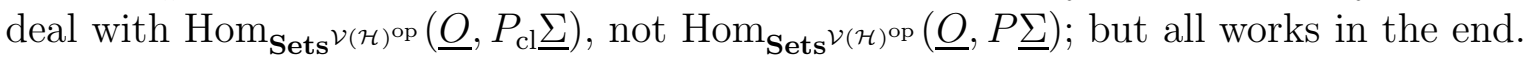

To prove that $\ulcorner\iota\urcorner: \underline{O} \rightarrow P_{\mathrm{cl}} \underline{\Sigma}$ is monic, it suffices to show that the map $\left\ulcorner_{\iota}\right\urcorner_{V}$ : $\underline{O}_{V} \rightarrow P_{\mathrm{cl}} \underline{\Sigma}_{V}$ is injective at all stages $V$. This is a straightforward exercise and the details will not be given here.

The conclusion of this exercise is that, since $\ulcorner\iota\urcorner: \underline{O} \rightarrow P_{\mathrm{cl}} \underline{\Sigma}$ is monic, the truth sub-objects $\underline{\mathbb{T}}^{|\psi\rangle}$ of $\underline{O}$ can also be regarded as sub-objects of $P_{\mathrm{cl}} \underline{\Sigma}$, and hence the truth value assignment in (4.28) is well-defined.

Finally then, for any given quantum state $|\psi\rangle$ the basic proposition " $A \varepsilon \Delta$ " can be assigned a generalised truth value $\nu(A \varepsilon \Delta ;|\psi\rangle)$ in $\Gamma \underline{\Omega}$, where $\tau:=\operatorname{Sets}^{\mathcal{V}(\mathcal{H})^{\text {op }}}$ is the topos of presheaves over $\mathcal{V}(\mathcal{H})$. This is defined at each stage/context $V$ as

$$
\begin{aligned}
\nu(A \varepsilon \Delta ;|\psi\rangle)_{V} & :=\nu\left(\ulcorner\delta(\hat{E}[A \in \Delta])\urcorner \in \underline{\mathbb{T}}^{|\psi\rangle}\right)_{V} \\
& =\left\{V^{\prime} \subseteq V \mid\ulcorner\delta(\hat{E}[A \in \Delta])\urcorner_{V^{\prime}} \in \underline{\mathbb{T}}_{V^{\prime}}^{|\psi\rangle}\right\} .
\end{aligned}
$$

\section{Conclusion}

In this, the second in our series of papers on topos theory and physics, we have started the development of a topos representation of quantum theory. In the first half of the 
paper, we have shown how propositions can be represented by clopen sub-objects of the spectral presheaf, $\underline{\Sigma}$, of the quantum theory. This is equivalent to finding a topos representation of the propositional language, $\mathcal{P} \mathcal{L}(S)$, that was discussed in paper I [1. A key ingredient in this representation is the concept of daseinisation, due to de Groote. By using this operation, a projection operator $\hat{E}[A \in \Delta]$ can be mapped to a global element $\delta(\hat{E}[A \in \Delta])$ of the outer presheaf $\underline{O}$, and, with the aid of the monic $\underline{O} \rightarrow P_{\mathrm{cl}} \underline{\Sigma}$, thereby to a (clopen) sub-object of the state object $\underline{\Sigma}$.

As was emphasised in the Introduction, this piece of work is something of a sideline in regard to the main programme, which is to find representations of the local language $\mathcal{L}(S)$. However, it is a satisfying (for us) completion of the earlier work on quantum theory and topoi.

But it is also a useful example with which to discuss the general problem of what lies 'inside' a language, and what lies 'outside'. In the case of the language $\mathcal{P} \mathcal{L}(S)$, it is clear that most 'symbols' in the theory lie outside. This includes (i) the topos; (ii) the physical quantities, $A$, and subsets, $\Delta \subseteq \mathbb{R}$, in the primitive propositions " $A \varepsilon \Delta$ "; and (iii) the state object whose sub-objects provide the Heyting algebra in which $\mathcal{P} \mathcal{L}(S)$ is represented.

On the other hand, in a representation of the language $\mathcal{L}(S)$, the only entity that necessarily lies outside the scope of the language, is the topos in which it is represented. As we will see in the next paper, III, the most general propositions in the $\mathcal{L}(S)$-theory are represented by the sub-objects $A_{\phi}^{-1}(\Xi)$, where $\Xi$ is any sub-object of the quantityvalue object $\mathcal{R}_{\phi}$ [2]. In this form, all the important physical ingredients in the theory have linguistic precursors in the language $\mathcal{L}(S)$ (with the exception of the topos $\tau_{\phi}$ ). It is this 'internal' language that would be used to manipulate propositions 'about the world' in a theory of this type.

The second part of the present paper is concerned with the idea of 'truth objects'. These play a central role in both the $\mathcal{P} \mathcal{L}(S)$ and the $\mathcal{L}(S)$-representations, and are the closest analogue there is to the notion of a micro-state in classical physics. It is likely that most topos-based theories of physics will use these objects because of the anticipated absence of microstates; classical physics is, of course, an exception.

The discussion of truth objects in Section 4.2 was formulated in terms of the language $\mathcal{L}(S)$, which gives a clear way of understanding the two types of 'state': microstates (global elements of the state object $\Sigma_{\phi}$ ), and truth objects (global elements of the power object $P\left(P \Sigma_{\phi}\right)$ ). However, the actual quantum-theory truth objects used in Section 4.2 are the ones given in the original papers on toposifying quantum theory, and no work has been done on them since then.

This is clearly an area in which further research is necessary. In particular, we would like to know if there are any generic properties of a truth object as an element in $P\left(P \Sigma_{\phi}\right)$; i.e., is there a theory of such things, or can any element of $P\left(P \Sigma_{\phi}\right)$ serve as one? This is related to a question that arises naturally when considering the specific truth objects, $\mathbb{T}^{|\psi\rangle}$, in quantum theory: namely what, if anything, can be said about the object $\underline{\mathbb{T}}^{\alpha|\psi\rangle+\beta|\phi\rangle}$ in regard to the objects $\underline{\mathbb{T}}^{|\psi\rangle}$ and $\underline{\mathbb{T}}^{|\phi\rangle}$ ? Or, to put it another way, 
is there an analogue for truth objects of the superposition of states? This is a very important subject for future research.

Acknowledgements This research was supported by grant RFP1-06-04 from The Foundational Questions Institute (fqxi.org). AD gratefully acknowledges financial support from the DAAD.

This work is also supported in part by the EC Marie Curie Research and Training Network "ENRAGE" (European Network on Random GEometry) MRTN-CT-2004005616 .

\section{References}

[1] A. Döring and C.J. Isham. A topos foundation for theories of physics: I. Formal languages for physics. (2007).

[2] A. Döring and C.J. Isham. A topos foundation for theories of physics: III. Quantum theory and the representation of physical quantities with arrows $\breve{\delta}^{o}(A): \underline{\Sigma} \rightarrow \underline{\mathbb{R}^{\succeq}}$. (2007).

[3] A. Döring and C.J. Isham. A topos foundation for theories of physics: IV. Categories of Systems. (2007).

[4] S. Kessari. Affine histories in quantum gravity: Introduction and the representation for a cosmological model. Classical and Quantum Grav. 24, 1303-1329, (2007).

[5] J.L. Bell. Toposes and Local Set Theories. Clarendon, Oxford (1988).

[6] R. Goldblatt. Topoi: The Categorial Analysis of Logic. North-Holland, London (1984).

[7] R.V. Kadison and J.R. Ringrose. Fundamentals of the Theory of Operator Algebras, Volume 1: Elementary Theory. Academic Press, New York (1983).

[8] A. Döring. Kochen-Specker theorem for von Neumann algebras. Int. J. Theor. Phys. 44, 139-160, (2005).

[9] S. Kochen and E.P. Specker. The problem of hidden variables in quantum mechanics. Journal of Mathematics and Mechanics 17, 59-87, (1967).

[10] H.F. de Groote. Observables. arxiv.org/abs/math-ph/0507019, (2005).

[11] Hans F. de Groote. Observables I: Stone spectra. arxiv.org/abs/math-ph/0509020, (2005). 
[12] H.F. de Groote. On a canonical lattice structure on the effect algebra of a von Neumann algebra. arxiv.org/abs/math-ph/0410018 v2, (2004).

[13] C.J. Isham and J. Butterfield. A topos perspective on the Kochen-Specker theorem: I. Quantum states as generalised valuations. Int. J. Theor. Phys. 37, 26692733, (1998).

[14] C.J. Isham and J. Butterfield. A topos perspective on the Kochen-Specker theorem: II. Conceptual aspects, and classical analogues. Int. J. Theor. Phys. 38, 827-859, (1999).

[15] J. Hamilton, J. Butterfield and C.J. Isham. A topos perspective on the KochenSpecker theorem: III. Von Neumann algebras as the base category. Int. J. Theor. Phys. 39, 1413-1436, (2000).

[16] J. Butterfield and C.J. Isham. A topos perspective on the Kochen-Specker theorem: IV. Interval valuations. Int. J. Theor. Phys. 41, 613-639, (2002). 